NBER WORKING PAPER SERIES

\title{
PREMIUM FOR HEIGHTENED UNCERTAINTY: EXPLAINING PRE-ANNOUNCEMENT MARKET RETURNS
}

\author{
Grace Xing $\mathrm{Hu}$ \\ Jun Pan \\ Jiang Wang \\ Haoxiang Zhu
}

Working Paper 25817

http://www.nber.org/papers/w25817

\author{
NATIONAL BUREAU OF ECONOMIC RESEARCH \\ 1050 Massachusetts Avenue \\ Cambridge, MA 02138 \\ May 2019, Revised March 2021
}

An earlier draft of this paper was circulated under the title "Premium for Heightened Uncertainty: Solving the FOMC Puzzle." We are grateful to Brad Barber, Ricardo Caballero, Peter Carr, Zhanhui Chen, Ing-Haw Cheng, Darrell Duffie, Ken French, Valentin Haddard, Toomas Laarits, David Lucca, Ian Martin, Annette Vissing-Jorgensen, Clara Vega, Kumar Venkataraman, Jessica Wachter, as well as seminar participants at the 2019 NBER Asset Pricing Program Spring Meeting, the 2019 ABFER Annual Meeting, the 2019 China International Conference in Finance, the 2019 Eastern Conference on Financial Mathematics, the 2019 Summer Institute in Finance, the 2020 AFA annual meeting, Tsinghua University, Shanghai Jiao Tong University, Peking University, Chinese University of Hong Kong, Cheung Kong Graduate School of Business, Southern Methodist University, Dartmouth College, Federal Reserve Board, Peking University HSBC Business School, and Penn State University for comments. We thank Meiling Chen and Zhe Geng for research assistance. The views expressed herein are those of the authors and do not necessarily reflect the views of the National Bureau of Economic Research.

NBER working papers are circulated for discussion and comment purposes. They have not been peerreviewed or been subject to the review by the NBER Board of Directors that accompanies official NBER publications.

(c) 2019 by Grace Xing Hu, Jun Pan, Jiang Wang, and Haoxiang Zhu. All rights reserved. Short sections of text, not to exceed two paragraphs, may be quoted without explicit permission provided that full credit, including $\odot$ notice, is given to the source. 
Premium for Heightened Uncertainty: Explaining Pre-Announcement Market Returns

Grace Xing Hu, Jun Pan, Jiang Wang, and Haoxiang Zhu

NBER Working Paper No. 25817

May 2019, Revised March 2021

JEL No. G12

\begin{abstract}
We find large overnight returns, with no abnormal variance, before the release of nonfarm payrolls, ISM, and GDP, similar to the pre-FOMC returns. To explain this common pattern, we propose a two-risk model with the uncertainty about the magnitude of the impending news' market impact as an additional risk, and link the pre-announcement return directly to the accumulation of heightened uncertainty and its later resolution prior to the announcement. We empirically test and verify the model's distinct predictions on the joint intertemporal behavior of return, variance, and particularly VIX - a gauge of impact uncertainty by our model, surrounding macroeconomic announcements.

Grace Xing $\mathrm{Hu}$

University of Hong Kong

818 K.K.Leung

Hong Kong

gracexhu@hku.hk

Jun Pan

Shanghai Advanced Institute of Finance

Shanghai Jiao Tong University

Shanghai, China

junpan@saif.sjtu.edu.cn

Jiang Wang

MIT Sloan School of Management

100 Main Street, E62-614

Cambridge, MA 02142

and NBER

wangj@mit.edu

Haoxiang Zhu

MIT Sloan School of Management

100 Main Street, E62-623

Cambridge, MA 02142

and NBER

zhuh@mit.edu
\end{abstract}




\section{Introduction}

Macroeconomic announcements are among the most important news events for the stock market. To the extent that these announcements bring aggregate risk to the market, they should be associated with a higher expected return, independent of their directional impact. Indeed, Savor and Wilson (2013) document significant positive stock market returns on days of well-known macroeconomic announcements, including the consumer price index, producer price index, employment figures, and the Federal Open Market Committee (FOMC) decisions. Lucca and Moench (2015) further show that these returns in fact arise mainly from the FOMC announcements. More importantly, they find that such returns are realized before the actual announcement, with no significant increase in the conventional risk measures such as return variance. Interestingly, post announcement, market returns are on average small and insignificant, despite the high variances it causes.

Against this backdrop, we document in this paper the presence of large pre-announcement returns ahead of a number of other important macroeconomic announcements, including nonfarm payrolls (NFP), the Institute for Supply Management's manufacturing index (ISM), and gross domestic product (GDP). From September 1994 to May 2018, the pre-announcement returns for NFP, ISM, and GDP are on average $10.1 \mathrm{bps}, 9.1 \mathrm{bps}$, and $7.5 \mathrm{bps}$, respectively, and all statistically significant. Using S\&P 500 index futures, these pre-announcement returns are calculated from the close of the previous trading day at $4 \mathrm{pm}$ to 5 minutes before the respective announcements, which are pre-scheduled at 8:30 am for NFP and GDP and 10 am for ISM. Effectively, the pre-announcement returns documented in our paper are realized mostly overnight - a key reason why this empirical fact has been missed by early studies including Lucca and Moench (2015). Benchmarked against the average overnight return of 0.69 bps for non-announcement days, the pre-announcement returns documented in our paper are large economically, and comparable to that of the pre-FOMC drift. ${ }^{1}$ Post announcement, the average returns for NFP, ISM, and GDP are small and insignificant, while exhibiting large variances, similar to the post-announcement patterns for FOMC.

Emerging from these findings is the rather intriguing realization that, common to the

\footnotetext{
${ }^{1}$ The pre-FOMC return, also calculated from the previous day's close to 5 minutes before the announcement, is on average $27.1 \mathrm{bps}$ during our sample period. This is lower than the 49 bps reported in Lucca and Moench (2015) for two reasons. First, our pre-announcement window starts from $4 \mathrm{pm}$ on the previous day, shorter than their 24-hours window. Second, we update their sample period to include the post-2011 period, when the pre-FOMC drift turned weaker. As we show later, while the pre-announcement returns for NFP, ISM and GDP are smaller than that for FOMC on event basis, they are actually larger on annual basis since there are more of them within a year.
} 
market-moving announcements such as NFP, ISM, GDP, and FOMC, there exists a unique risk-and-return pattern - large pre-announcement returns with small variances, followed by small post-announcement returns with large variances. Using the return-to-variance ratio as a measure of market price of risk, which is insensitive to time scale, we can infer that more than one aggregate risk is driving the stock market return in the pre- and postannouncement windows. In particular, since the same risk carries the same risk premium, a single risk cannot generate the significantly different return-to-variance ratios before and post the announcement, even allowing for pre-announcement news leakage. ${ }^{2}$

Motivated by these observations, we develop a parsimonious two-risk model to capture the different risks surrounding a macroeconomic announcement and the resulting risk-andreturn pattern. In our model, the news is given by a random shock $\varepsilon$, which is directional in nature and has zero mean $(\mathrm{E}[\varepsilon]=0)$. The magnitude of the news' impact on the market is given by $\sigma$, which is non-directional (hence always positive). The total market impact of the news is then given by the product of these two components, $\sigma \varepsilon$. In general, $\sigma$ is uncertain, which gives rise to the second risk concerning the announcement, the "impact uncertainty." While the news risk itself is fully resolved at the announcement, its "impact uncertainty" is resolved before the announcement. ${ }^{3}$ Central to our model is the presence of this second risk concerning $\sigma$, whose variability is determined by its own volatility, given by a parameter $\lambda$. Intuitively, depending on the realization of $\sigma$, the same news $\varepsilon$ could have substantially different market impact, with parameter $\lambda$ capturing the level of this uncertainty - when $\lambda$ is large, the impact uncertainty is large, giving rise to heightened uncertainty in anticipation of a major macroeconomic announcement.

Each of these two risks, news risk vs impact uncertainty, carries its own premium and impacts the price dynamics differently. In our model, we show that when $\lambda$ is sufficiently high, the impact uncertainty carries a higher risk premium in equilibrium than the news risk. Moreover, the premiums for the two risks are realized over different time windows owing to the timing difference of their respective rise and resolution. Throughout of the paper,

\footnotetext{
${ }^{2}$ Theoretically speaking, a single risk factor can lead to different return-to-variance ratios for different time periods if its resolution is nonlinear overtime and return variance fails to properly measure risk. Such a nonlinearity can arise from various causes ranging from risk dynamics itself to market imperfections including information asymmetry. Given the short horizons for the pre- and post-announcement periods and the public nature of the information, it is unlikely that the issue of nonlinearity is substantial to explain the empirical magnitudes shown later in the paper.

${ }^{3}$ Our model also allows part of the news risk $\varepsilon$ to be resolved prior to the announcement to accommodate the possibility of news leakage prior to the announcement. The use of "uncertainty" here for the $\sigma$ risk is only in an intuitive sense, to differentiate from the news risk itself.
} 
in both model development and empirical test, we focus on three distinct time windows surrounding an announcement: 1) the accumulation period is when, in anticipation of a scheduled announcement, the impact uncertainty builds up; 2) the pre-announcement period is when the impact uncertainty resolves prior to the announcement and the premium for impact uncertainty is realized; and 3) the post-announcement period is when the news risk is fully resolved. To directly connect to data, our model further provides distinctive empirical measures for the two risks - the magnitude of the news risk is properly captured by return variance, while the magnitude of the impact risk $\lambda$ is found to be linked directly to the market price of a variance swap (i.e., $\mathrm{VIX}^{2}$ ).

Depending on the magnitude of impact uncertainty $\lambda$, the model leads to the following predictions on the joint intertemporal dynamics of return, variance, and VIX. First, in the absence of impact uncertainty $(\lambda=0)$, the return-to-variance ratio should be the same for both the pre- and post-announcement periods, regardless of whether or not there is partial resolution of the news risk before the actual announcement. This is simply because the same risk carries the same risk premium. Second, in the presence of heightened impact uncertainty ( $\lambda$ large), both the pre-announcement return and return-to-variance ratio will be higher than their post-announcement counterparts. In addition, the high pre-announcement return will be accompanied by a large drop in VIX, reflecting the resolution of impact uncertainty. Third, a large increase in VIX during the accumulation period will be accompanied by a drop in price, and then followed by a large pre-announcement return, a large return-tovariance ratio, and a large decrease in VIX. This signature pattern - a gradual built-up of heightened uncertainty followed by its rapid resolution prior to the announcement - is uniquely linked to the key underlying mechanism of our model.

Taking the model to the data, we examine the model's predictions on the joint behavior of return, variance, and VIX over the three time periods, defined empirically as follows. The post-announcement period begins 5 minutes before the announcement and ends at the announcement day's close at $4 \mathrm{pm}$. The pre-announcement period begins at the previous day's close and ends at the beginning of the post-announcement window. The accumulation period begins six days before the announcement and ends at the beginning of the preannouncement window. ${ }^{4}$ Our empirical results can be summarized as follows.

\footnotetext{
${ }^{4}$ One empirical challenge in defining the accumulation window is that we do not know exactly when uncertainty starts to build up, which may vary substantially across announcements. Using any fixed window across all announcements inevitably introduces noises in our measurement and weakens our results. Moreover, the pre-scheduled nature of macroeconomic releases allows investors to trade well in advance, which in turn mask the real market impact over a relatively long time window.
} 
Pre-announcement returns: According to the model, the pre-announcement period is when the premium for heightened uncertainty is realized. Consistent with this prediction, we find large pre-announcement returns for the four major macroeconomic announcements, NFP, ISM, GDP and FOMC. Pooling the four announcements together, the average preannouncement return is $5.66 \%$ annually, realized over the pre-announcement windows of a mere 44 announcements per year. ${ }^{5}$ Excluding FOMC, whose pre-announcement return has been previously documented by Lucca and Moench (2015), the average pre-announcement return remains important and significant at $3.41 \%$ per year, realized over the pre-announcement windows, mostly overnight, of 36 announcements per year. By comparison, the average annual return over the same sample period is $9.10 \%$, realized over 252 days per year. These results, measured across four different types of macroeconomic announcements, provide a compelling evidence that the presence of heightened uncertainty is common to important macroeconomic announcements. Compared with NFP, ISM, and GDP, the single-day impact of FOMC announcement might be larger, but the essence of its pre-announcement return is the same and not unique. Cumulatively, NFP, ISM and GDP actually yield larger preannouncement returns than FOMC, 3.41\% vs. 2.25\% per year respectively, simply because they have more announcements.

Our examination of the pre-announcement returns across a broad spectrum of macroeconomic indicators also reveals a substantial heterogeneity across these indicators. In the context of our model, the varying levels of pre-announcement return reflect the varying magnitudes of impact uncertainty. Some macroeconomic indicators, such as FOMC, NFP, ISM, and GDP, exhibit significant pre-announcement returns on average, indicating strong heightened uncertainty brought upon by these announcements to the market, while some indicators are found to have insignificant pre-announcement returns, indicating weak impact uncertainty. Indeed, lining up macroeconomic indicators by their pre-announcement returns, the ranking is consistent with their relative importance, both intuitively perceived by market participants and empirically documented by prior literature.

Return-to-risk ratios: We find the return-to-variance ratio to be markedly different between the post- and pre-announcement periods, a clear challenge to the single-risk model. The return-to-variance ratio, which is invariant over time scale, is 35.53 and strongly significant during the pre-announcement period, and a mere 2.34 and insignificant during the

\footnotetext{
${ }^{5}$ There are 12 pre-scheduled announcement days per year for NFP, ISM and GDP, respectively, and 8 announcement days per year for FOMC.
} 
post-announcement period. ${ }^{6}$ This result, robust with and without FOMC as part of the macroeconomic announcements as well as over different subperiods, also contradicts the explanation that information leakage with only the news risk might be behind the large preannouncement return. In the context of our setting, having only one news risk, regardless of leakage, cannot explain the differing return-to-variance ratios across the two time periods.

Resolution of impact uncertainty: One important implication of our model is that the pre-announcement return arises out of the resolution of heightened uncertainty, which can be captured by the reduction in VIX during the pre-announcement period. Exploring this connection, we use the pre-announcement reduction in VIX to sort announcement days into groups of high and low resolution of uncertainty. As predicted by the model, we find significantly larger pre-announcement returns for the high group and insignificant and negative pre-announcement returns for the low group. Given the well-known negative correlation between market returns and changes in variance, we further double-sort announcement days using pre-announcement reductions in VIX as well as variance, and find the result to be driven by VIX. Specifically, using the macroeconomic announcements of NFP, ISM, GDP, and FOMC, we find that days of high reduction in VIX are associated with large pre-announcement returns, averaged at 76.6 bps and 64.8 bps, respectively, for groups of high and low reduction in variance. By contrast, days of low reduction in VIX yield pre-announcement returns of $-5.0 \mathrm{bps}$ and $-8.8 \mathrm{bps}$, respectively, for groups of high and low reduction in variance. Given that VIX and variance contain different information in our model, with VIX uniquely linked to the magnitude of impact uncertainty, this result further strengthens the connection between the presence of impact uncertainty and the pre-announcement return.

Heightened uncertainty and it risk premium: The accumulation period is when heightened uncertainty arises in anticipation of the impending announcement. In the context of our model, the higher the impact uncertainty $\lambda$, the stronger the built-up during the accumulation period, which leads to lower returns in the same period and larger preannouncement returns. Following this prediction, we use the increase in VIX during the accumulation period to sort announcements of the four major macroeconomic indicators (NFP, ISM, GDP, and FOMC) into high and low impact uncertainty groups. As predicted by the model, we find that the accumulation periods in the high group indeed exhibit low

\footnotetext{
${ }^{6} \mathrm{With}$ an average daily return of $4 \mathrm{bps}$ and daily variance of $1 \mathrm{bp}$ observed for the aggregate market, the return-to-variance ratio is benchmarked at 4 for an average day in the US stock market.
} 
contemporaneous returns, which are further followed by significantly higher returns and steeper VIX reductions in the pre-announcement period. This predictive result, driven by the fact that one single quantity $\lambda$, as a measure of uncertainty, has implications for both time periods, offers rather compelling evidence that the pre-announcement return is indeed a premium for heightened uncertainty.

The fact that heightened uncertainty leads to large market return is an important and rather unique prediction of our model. To further strengthen this novel empirical fact, we use the change in VIX over the six-day accumulation window to predict the pre-announcement return for the four major macroeconomic indicators. Indeed, we find that changes in VIX during the accumulation period can positively predict pre-announcement returns with an adjusted R-squared of 3.13\%. For such high-frequency predictive regressions of daily market return, this level of predictive power is rather large. Moreover, with the exception of FOMC, a large fraction of the pre-announcement returns are in fact realized overnight.

Unanticipated heightened uncertainty: Besides scheduled announcements, heightened uncertainty can also be triggered unexpectedly. In the context of our model, a large spike in VIX on a single day can be viewed as a condensed, sped-up version of the slow accumulation of VIX in anticipation of a scheduled announcement. Just as a large accumulation in VIX triggered by an anticipated announcement leads to a higher subsequent return, so should a sudden spike in VIX be followed by a large subsequent return. This is indeed what we find in the data. Focusing on non-announcement days, we identify days of unanticipated heightened uncertainty using sudden and large increases in VIX. Consistent with our model's prediction, we find that such heightened VIX days are followed by large next-day market returns, with magnitudes comparable to the pre-announcement returns.

In parallel to the scheduled announcements, we repeat the same predictive regression for non-announcement days, also using changes in VIX over a six-day window to predict the nextday market returns. Interestingly, we find predictability only for those non-announcement days with heightened VIX and the adjusted R-squared of the predictive regression is $2.34 \%$, comparable to that for the scheduled announcements. For all other non-announcement days, changes in VIX cannot predict the next-day returns and the R-squared is essentially zero.

The finding that unanticipated heightened uncertainty also leads to large market returns serves as an out-of-the-sample test of our model, further strengthening its empirical support. After all, regardless of its origin, heightened uncertainty, anticipated as well as unanticipated, brings risk as well as risk premium to the market, and this is the essence of our model. 


\section{Literature and Discussion}

Our paper is most closely related to the empirical literature studying the stock impact of macroeconomic announcements. Savor and Wilson (2013) provide some of the earliest evidence linking the risk from macroeconomic announcements to stock-market risk premium. Lucca and Moench (2015) are the first to identify the large market returns prior to FOMC announcements. ${ }^{7}$ Following this literature, we are the first to uncover the significant overnight return prior to the release of a number of other macroeconomic indicators including NFP, ISM and GDP. Proper inclusion of the overnight window in the pre-announcement period sets our empirical findings apart from those of Lucca and Moench (2015), which, excluding the overnight window, report small and insignificant pre-announcement returns for non-FOMC macroeconomic indicators. ${ }^{8}$ Moreover, instead of focusing exclusively on the pre-announcement period, we also examine the risk-and-return patterns surrounding the announcements. In particular, it is important to show that there is no abnormal return after the announcement, from 5 minutes before the announcement to the day's closing, despite a high return variance over the same period.

Our paper also contributes to the theoretical modeling of the asset pricing implications of the market-moving macroeconomic announcements. Combining the results for FOMC from Lucca and Moench (2015) and for NFP, ISM and GDP from our paper, there is compelling evidence that the macroeconomic "announcement-day" returns in the U.S. stock market are predominantly pre-announcement returns. Motivated by this observation, our model departs from other models in the literature that study conditions under which macroeconomic announcement generates a positive stock return, such as Ai and Bansal (2018) and Wachter and Zhu (2019). These studies do not distinguish pre-announcement and post-announcement returns. Our evidence reveals that they behave markedly differently: the pre-announcement part dominates in average return and has a much higher return-to-variance ratio than the post-announcement counterpart. Therefore, a key contribution of ours is the explicit modeling of the pre-announcement period through the lens of impact uncertainty and the empirical

\footnotetext{
${ }^{7}$ See also Gilbert, Kurov, and Wolfe (2018) and Lucca and Moench (2018). Brusa, Savor, and Wilson (2020) and Guo, Jia, and Sun (2019) have examined pre-announcement returns for other major central banks' monetary policy decisions and found mixed results. For a set of non-FOMC macroeconomic announcements, $\mathrm{Ai}$ and Bansal (2018) also report positive announcement-day returns when pooling together the pre- and post-announcement returns, without separating them. Ernst, Gilbert, and Hrdlicka (2019) have also studied the announcement-day returns for a range of other macroeconomic indicators and their potential connection with sample selection.

${ }^{8} \mathrm{Ai}$ and Bansal (2018) also report insignificant pre-announcement returns on a set of non-FOMC macroeconomic indicators, excluding the overnight window from the pre-announcement period.
} 
characterization of the joint dynamics of returns and VIX during the accumulation and preannouncement periods.

By extending our empirical analysis beyond FOMC and establishing a theoretical foundation common to all important macroeconomic announcements, we add discipline as well as richness to the literature that focuses exclusively on the pre-FOMC drift. For example, Cieslak, Morse, and Vissing-Jorgensen (2019) suggest that the large pre-FOMC drift is the result of news leakage prior to the announcement of unexpectedly accommodating monetary policy. To study the pricing implications of news leakage, our model allows part of the news risk to be resolved prior to the announcement and shows that gradual resolution of the news risk alone cannot explain the sharp difference in return and variance behavior between the pre- and post-announcement periods. ${ }^{9}$ Because the pre-announcement revelation of impact uncertainty may be broadly interpreted as a form of "leakage" or learning, our model is not inconsistent with a general notion of learning or leakage. However, whatever is revealed pre-announcement must be distinct from the announced news itself to generate a much larger return-risk ratio as seen in the data. From this perspective, the leakage of the actual announcement itself as the only relevant information cannot be the full explanation of the pre-announcement drift. In a paper subsequent to ours, Laarits (2020) proposes an additional state variable for the economy (good or bad), whose revelation before the announcement yields the pre-FOMC return. Like in our model, this state variable introduces a second source of risk. However, unlike ours, his model does not fully explore its implications for the joint intertemporal behavior of return and risk around the announcement. Moreover, while his focus is on the pre-FOMC drift alone, our model addresses the pre-announcement return common to all important macroeconomic announcements.

The fact that VIX emerges as a measure for impact uncertainty in our model connects our paper to the rich literature on the dynamic relationship between return, variance, and VIX. Generally, in the presence of multiple risks, VIX can merge as an instrument for risks in addition to the directional payoff risk, which can be captured by return variance. ${ }^{10}$ The

\footnotetext{
${ }^{9}$ In standard rational expectations settings, He and Wang (1995) and Jiang, Pan, and Qiu (2019) show that informed trading can lead to pre-announcement realization of the risk premium as private information gets incorporated into the price. However, as shown in Bernile, Hu, and Tang (2016) and Kurov, Sancetta, Strasser, and Wolfe (2019), evidence on informed trading, if any, is only detected 30 minutes before macroeconomic announcements. In addition, informed trading tend to bring more variance to the price due to the risk of adverse selection, lowering the return-to-variance ratio.

${ }^{10}$ In the option pricing literature, Pan (2002) shows that option prices contain information not only about the underlying return variance but also the risk premium for crash as well as variance risk. In a model of dynamic information acquisition, Han (2019) shows that VIX squared can emerge as an endogenous
} 
most salient empirical fact in this literature is the the contemporaneous negative correlation between returns and changes in volatility as studied by Black (1976), Christie (1982), French, Schwert, and Stambaugh (1987), Campbell and Hentschel (1992), Bekaert and Wu (2000) and $\mathrm{Wu}$ (2001). Given the volatility information embedded in option prices as well as VIX, evidence of this negative correlation has also been documented by Bakshi, Cao, and Chen (1997) and Pan (2002) using option prices, and by Dennis, Mayhew, and Stivers (2006) and Carr and Wu (2006) using VIX. Relating to this literature, we provide evidence that the magnitude of impact uncertainty surrounding the macroeconomic announcements is uniquely linked to VIX, not variance. In particular, we show that resolution of impact uncertainty during the pre-announcement window is captured by the reduction in VIX not variance. Another important empirical fact in this literature, as documented by Bollerslev, Tauchen, and Zhou (2009), is that variance premium - the difference between VIX ${ }^{2}$ and realized variance, can predict stock market returns at the intermediate quarterly horizon. We contribute to this literature by showing that changes in VIX can positively predict the nextday stock returns under scenarios of heightened uncertainty, either triggered unexpectedly or in anticipation of an impending macroeconomic announcement.

Our model is silent on what drives the heightening of impact uncertainty for certain announcements, its resolution and their timing. They may be linked to the exogenous flow of information to the economy and/or endogenous acquisition of information by investors. There is a an emerging literature that studies investor behavior prior to macroeconomic announcements, adding granular evidence for the resolution of uncertainty pre-announcement. ${ }^{11}$ We return to the discussion of these issues in the concluding section.

measure of asset's payoff uncertainty when noise trading brings another source risk. In the context of macroeconomic announcements, one can also try to construct other empirical measures of uncertainty. For example, for FOMC, Bauer, Lakdawala, and Mueller (2019) use the standard deviation of LIBOR as a proxy for monetary policy uncertainty, and find this measure of uncertainty declines substantially on the day of FOMC announcements. Fisher, Martineau, and Sheng (2018) find a positive correlation between VIX and their "macroeconomic attention index" based on news articles at the daily frequency.

${ }^{11}$ For example, Savaser (2011) finds that investors in the GBP/USD foreign exchange market submit significantly more stop-loss orders and take-profit orders between 3:30 am and 6:30 am on days with an impending 8:30 am U.S. macroeconomic announcement (including NFP and GDP) than non-announcement days. These orders are limit orders with given prices, so investors seem to have formed their views on the impact of the upcoming announcements during the overnight period, coinciding with the high overnight stock return. Benamar, Foucault, and Vega (2020) find that clicks on news articles related to NFP increases significantly from 4 am to the announcement time of 8:30 am, indicating that learning intensifies approaching the announcement. 


\section{A Model of Risks and Returns around Announcements}

In this section, we develop a simple asset pricing model, which captures two types of risks concerning a macro announcement. One risk is about a directional news on the economy; the other risk, also referred to as uncertainty for distinction, is about the magnitude of the news' impact on asset payoffs. In general, these two risks generate different risk premiums and return variability. When the resolution of these two risks occur at different times, it will lead to rich intertemporal return patterns. We demonstrate that the resolution of heightened uncertainty brought by a news, followed by the resolution of the news itself can generate the return and risk patterns similar to those around macroeconomic announcements.

\subsection{Setup}

For simplicity, we consider an economy with three dates, $t=0,1,2$.

\section{Securities Market}

There is frictionless securities market, which include two primitive securities, a bond and a stock. Each unit of the bond yields a terminal sure payoff of 1 at $t=2$. Each share of the stock pays a terminal risky payoff $D$ at $t=2$. $D$ is given by:

$$
D=\bar{D}+\sigma \varepsilon=\bar{D}+\sigma\left(\varepsilon_{1}+\varepsilon_{2}\right),
$$

where $\bar{D}$ is a positive constant, and $\sigma, \varepsilon_{1}$, and $\varepsilon_{2}$ are independent random variables. We assume that $\sigma$ has a positive mean while $\varepsilon_{1}$ and $\varepsilon_{2}$ have zero means. Without loss of generality, the variances of $\varepsilon_{1}$ and $\varepsilon_{2}$ are assumed to sum to 1 (i.e., $\varepsilon$ has a variance of 1 ). Thus, $\bar{D}$ gives the expected dividend and $\sigma^{2}$ gives the variance of dividend.

In the context of this paper, $\varepsilon_{1}$ and $\varepsilon_{2}$ are two components of a market-moving news $\varepsilon$, and $\sigma^{2}$ captures the size of its impact on asset payoffs. All three variables are unknown to the market ex ante but revealed over time gradually. In particular, heightened uncertainty prior to the news is represented by a high ex ante variance of $\sigma^{2}$.

Both the bond and the stock are traded in the market at dates 0,1 and 2 . We will use the bond as the numeraire and denote the price of the stock at date $t$ as $P_{t}, t=0,1,2$. Since the bond is the numeraire, its price will remain at one and its return is always zero.

For tractability, we further assume that $\sigma^{2}$ follows an exponential distribution with location parameter $\lambda_{0} \geq 0$ and scale parameter $\lambda \geq 0$, where $\lambda_{0}$ and $\lambda$ are known constants. That is, $\sigma^{2}$ has the support $\left[\lambda_{0}, \infty\right)$, and $\sigma^{2}-\lambda_{0}$ follows an exponential distribution with 
variance $\lambda^{2}$. In addition, $\varepsilon_{1}$ and $\varepsilon_{2}$ follow normal distributions with mean zero and variances $\delta$ and $1-\delta$, respectively, where $\delta \in[0,1]$ is also a known constant.

Let $\mathrm{E}_{t}[\cdot]$ and $\mathrm{V}_{t}[\cdot]$ denote the conditional mean/expectation and variance of a random variable at time $t, t=0,1$, respectively. The conditional mean and variance at $t=0$ also give the unconditional mean and variance, respectively, for which we drop the time subscript for convenience. We then have:

$$
\mathrm{E}\left[\sigma^{2}\right]=\lambda_{0}+\lambda, \quad \mathrm{V}\left[\sigma^{2}\right]=\lambda^{2} .
$$

Thus, a larger value of $\lambda$ corresponds to a higher unconditional mean and variance of $\sigma^{2}$. For comparative statics, we can hold the mean of $\sigma^{2}$ constant and increase its variance by increasing $\lambda$ and decreasing $\lambda_{0}$ by the same amount. Thus, increasing $\lambda$ corresponds to increasing the variance of $\sigma^{2}$ or uncertainty.

\section{Investors}

There is a unit mass of identical, infinitesimal, and competitive investors, who are endowed with zero unit of the bond and one share of the stock. In addition, we assume that all investors have CARA utility over their terminal wealth:

$$
-\exp \left\{-\alpha W_{2}\right\}
$$

where $\alpha>0$ is the risk aversion coefficient and $W_{2}$ is the wealth at $t=2$.

For the model to be well-defined, the following parameter condition is needed:

$$
\lambda<\frac{2}{\alpha^{2}}
$$

Since the mean of $\sigma^{2}$ will be held constant, condition (4) imposes upper bounds on its variance, i.e., uncertainty. From now on, we assume (4) always holds without repeating it.

\section{Time Line}

The time line for the economy is summarized as follows:

$t=0$ : Investors know about the underlying parameters of the economy, $\bar{D}, \lambda_{0}, \lambda$ and $\delta$, but observes none of $\sigma^{2}, \varepsilon_{1}$, and $\varepsilon_{2}$. Based on their probability distributions, investors trade the stock (against the bond) by submitting competitive demand functions, and the market clears at the equilibrium price $P_{0}$. 
$t=1$ : Investors observe $\sigma^{2}$ and $\varepsilon_{1}$, but not $\varepsilon_{2}$. We refer to the revelation of $\sigma^{2}$ as the resolution of uncertainty. In addition, in terms of the news itself, a fraction $\delta$ of its content also becomes known to the market before it is officially announced. Investors trade in the market again, yielding the equilibrium stock price $P_{1}$.

$t=2$ : The second component of the news $\varepsilon_{2}$ is realized, dividend $D$ is paid on the stock, and investors consume their terminal wealth.

The time line above describes how the two risks, one about the news itself and the other about its impact, arise and resolve over time. In particular, at $t=0$, the size of the news risk and impact risk, or uncertainty, are determined by $\lambda_{0}$ and $\lambda$. During the first period, i.e., by $t=1$, the magnitude of the news' impact, $\sigma^{2}$, is learned. In addition, part of the news' content, $\varepsilon_{1}$, is also leaked to the market. The amount of leakage is captured by $\delta$. For example, $\delta=0$ corresponds to the case of no leakage, and $\delta=1$ corresponds to the case of full leakage. By the end of second period, i.e., at $t=2$, the remaining part of the news, $\varepsilon_{2}$, is revealed to the market.

\section{Discussion}

Several comments are in order before we move on. First, our model is intentionally simple, aimed at capturing the two important risks concerning a macroeconomic news, the risk about the news realization itself and the uncertainty about its impact, and their intertemporal resolution. Our main goal is to show qualitatively how such a model can lead to the possible return and volatility dynamics observed in the data. The model can be extended to a full intertemporal setting and to allow richer dynamics for the two risks.

Second, since we mainly care about the price implications of the model, we have abstracted away from potential heterogeneity among investors and the actual trading between them. The model can allow different types of heterogeneity, such as heterogeneous endowment shocks, signals on $\sigma^{2}$ and $\varepsilon$, and their interpretations, while yielding similar pricing implications.

In addition, the timing for the resolution of the two risks is given exogenously in the model. It is possible that this resolution process is driven, at least partially, by the investors' information production process. Our model can be extended to endogenize the timing of the risk resolution by explicitly modeling investors' information production decisions. But this is beyond the scope of this paper. 
Finally, assumptions on probability distributions and investor preferences are made for tractability. Thus, our results are not meant to be restrictive but mainly illustrative.

\subsection{Equilibrium}

We solve the model backwards. Because investors are identical, we can solve the problem of a generic investor, without loss of generality. We denote by $W_{t}$ the wealth of a generic investor at the end of date $t$, and denote by $\theta_{t}$ the investor's demand of the risky asset at date $t$.

Solution for date 1. An investor's consumption at date 2 is:

$$
W_{2}=W_{1}+\theta_{1}\left(D-P_{1}\right)
$$

At date 1 , after $\sigma$ and $\varepsilon_{1}$ are known, the final dividend $D$ is normally distributed with mean $\bar{D}+\sigma \varepsilon_{1}$ and a known variance $(1-\delta) \sigma^{2}$. So the investor's optimization problem is:

$$
\begin{aligned}
J_{1} & =\max _{\theta_{1}} \mathrm{E}_{1}\left[-\exp \left\{-\alpha\left[W_{1}+\theta_{1}\left(D-P_{1}\right)\right]\right\}\right] \\
& =\max _{\theta_{1}}-\exp \left\{-\alpha\left[W_{1}+\theta_{1}\left(\bar{D}+\sigma \varepsilon_{1}-P_{1}\right)-\frac{1}{2} \alpha(1-\delta) \sigma^{2} \theta_{1}^{2}\right]\right\} .
\end{aligned}
$$

The investor's demand function is then given by:

$$
\theta_{1}=\frac{\mathrm{E}_{1}\left[D-P_{1}\right]}{\alpha \mathrm{V}_{2}\left[D-P_{1}\right]}=\frac{\bar{D}+\sigma \varepsilon_{1}-P_{1}}{\alpha(1-\delta) \sigma^{2}} .
$$

The demand function is easy to understand. It is proportional to the expected net payoff from the stock, given by the numerator, and inversely proportional to the risk aversion $\alpha$ and the variance of the stock's payoff $(1-\delta) \sigma^{2}$, which measures the its remaining risk over the second period, as shown in the denominator.

From the market clearing condition $\theta_{1}=1$, the equilibrium stock price at $t=1$ is:

$$
P_{1}=\left(\bar{D}+\sigma \varepsilon_{1}\right)-\alpha(1-\delta) \sigma^{2}
$$

The stock price has a simple interpretation. The first two terms, $\bar{D}+\sigma \varepsilon_{1}$, is the stock's expected payoff, given $\sigma$ and $\varepsilon_{1}$, which are known at $t=1$. The second term, $-\alpha(1-\delta) \sigma^{2}$, gives the risk discount on the price. It is proportional to $\alpha$, the risk aversion, and, more importantly, the remaining risk about the news, $\varepsilon_{2}$, which is $(1-\delta) \sigma^{2}$. 
Solution for date 0 . Substituting the equilibrium strategy at $t=1$ into $J_{1}$, we get:

$$
J_{1}=-\exp \left\{-\alpha\left[W_{0}+\theta_{0}\left(\bar{D}+\sigma \varepsilon_{1}-\alpha(1-\delta) \sigma^{2}-P_{0}\right)+\frac{1}{2} \alpha(1-\delta) \sigma^{2}\right]\right\},
$$

where we have also used $W_{1}=W_{0}+\theta_{0}\left(P_{1}-P_{0}\right)$.

Recall that at $t=0$, investors have an exponential distribution for $\sigma^{2}$ with $\lambda_{0}$ and $\lambda$ and a normal distribution for $\varepsilon_{1}$ with mean 0 and variance $\delta$. To calculate $\mathrm{E}\left[J_{1}\right]$, we take iterated expectations, first by conditioning on $\sigma$ :

$$
\begin{aligned}
\mathrm{E}\left[J_{1} \mid \sigma\right] & =-\exp \left\{-\alpha\left[W_{0}+\theta_{0}\left(\bar{D}-P_{0}\right)\right]+\alpha^{2}\left[\frac{1}{2}(1-\delta)-(1-\delta) \theta_{0}-\frac{1}{2} \delta \theta_{0}^{2}\right] \sigma^{2}\right\} \\
& \equiv-\exp \left\{-\alpha\left[W_{0}+\theta_{0}\left(\bar{D}-P_{0}\right)\right]+\alpha^{2} Q\left(\theta_{0}, \delta\right) \sigma^{2}\right\}
\end{aligned}
$$

where

$$
Q\left(\theta_{0}, \delta\right) \equiv \frac{1}{2}(1-\delta)-(1-\delta) \theta_{0}-\frac{1}{2} \delta \theta_{0}^{2},
$$

is a quadratic function of $\theta_{0}$ as the coefficient in front of $\sigma^{2}$ in the exponent. Taking expectations over $\sigma^{2}$ gives:

$$
\begin{aligned}
\mathrm{E}\left[J_{1}\right]=\mathrm{E}\left[\mathrm{E}\left[J_{1} \mid \sigma\right]\right] & =-e^{-\alpha\left[W_{0}+\theta_{0}\left(\bar{D}-P_{0}\right)\right]} \int_{\lambda_{0}}^{\infty} e^{-\alpha^{2} Q\left(\theta_{0}, \delta\right) x} \frac{1}{\lambda} e^{-\frac{x-\lambda_{0}}{\lambda}} d x \\
& =-e^{-\alpha\left[W_{0}+\theta_{0}\left(\bar{D}-P_{0}\right)\right]-\alpha^{2} Q\left(\theta_{0}, \delta\right) \lambda_{0}} \frac{1}{1+\alpha^{2} \lambda Q\left(\theta_{0}, \delta\right)},
\end{aligned}
$$

subject to the condition that

$$
1+\alpha^{2} Q\left(\theta_{0}, \delta\right)=1+\alpha^{2} \lambda\left[\frac{1}{2}(1-\delta)-(1-\delta) \theta_{0}-\frac{1}{2} \delta \theta_{0}^{2}\right]>0 .
$$

In equilibrium, $\theta_{0}=1$, so this condition becomes $\alpha^{2} \lambda<2$, which is simply (4).

To solve the equilibrium price, we first solve for the investors' stock demand. The corresponding first order condition is:

$$
\frac{d \mathrm{E}\left[J_{1}\right]}{d \theta_{0}}=0
$$

which gives the optimal $\theta_{0}$, as shown in the appendix. Substituting in $\theta_{0}=1$, the market clearing condition, we obtain the equilibrium stock price at date 0 :

$$
P_{0}=\bar{D}-\alpha\left(\lambda_{0}+\lambda\right)-\frac{\frac{1}{2} \alpha^{3} \lambda^{2}}{1-\frac{1}{2} \alpha^{2} \lambda} .
$$

The stock price $P_{0}$ given in (15) has a simple interpretation. Its first term is simply the stock's expected payoff at $t=0$. The next two terms then represent two separate discounts, 
for the two risks in the stock's payoff, $\varepsilon$ and $\sigma^{2}$, respectively. The first discount, $\alpha\left(\lambda_{0}+\lambda\right)$, is associated with the news risk. It is proportional to $\lambda_{0}+\lambda$, which is the expected variance of $D, \mathrm{E}\left[\sigma^{2}\right]$, arising from the news itself and its expected impact. The proportionality coefficient is simple $\alpha$, the risk aversion. It is important to note that this discount only depends on the mean of $\sigma^{2}$, which is $\lambda_{0}+\lambda$. Holding $\lambda_{0}+\lambda$ constant, this discount will not change even if $\lambda=0$ and there is no uncertainty.

The second discount, given by the third term in (15), is associated with impact uncertainty, which is captured by the variance of $\sigma^{2}, \lambda^{2}$. Indeed, this discount is independent of $\lambda_{0}$, but proportional to $\lambda^{2}$, as the numerator indicates. When there is no uncertainty, that is, when $\lambda=0$, this discount becomes zero. Holding $\mathrm{E}\left[\sigma^{2}\right]=\lambda_{0}+\lambda$ constant, this discount increases with $\lambda$. Furthermore, when $\lambda$ becomes sufficiently large, for example, when it is close to its upper limit $2 / \alpha^{2}$, this discount can be very large. This implies that a heightened uncertainty, as given by a large enough value of $\lambda$, will lead to a large discount on the current stock price. Moreover, the resolution of this uncertainty, when it occurs later, will lead to a large return, reflecting the corresponding risk premium for the uncertainty in $\sigma^{2}$.

The following proposition summarizes the equilibrium stock prices.

Proposition 1 The equilibrium stock price at dates 0 and 1 are given by (15) and (8), respectively. Moreover, the stock price at date 0 is decreasing in $\lambda_{0}$ and in $\lambda$.

\subsection{Uncertainty and VIX}

In order to test the model's implications, it is desirable to have measures that will allow us to capture the two separate risks in the model, $\mathrm{E}\left[\sigma^{2}\right]=\lambda_{0}+\lambda$ and $\mathrm{V}\left[\sigma^{2}\right]=\lambda^{2}$. $\mathrm{E}\left[\sigma^{2}\right]$ is easy to estimate empirically, since it is the expected variance of dividend (or return). What we hope to do is to find an observable variable that can capture the uncertainty about $\sigma^{2}$.

For this purpose, we consider a forward-looking variance swap, which pays $\left(D-P_{1}\right)^{2}$ at $t=2$. Its price, denoted by $v_{t}$, is given by:

$$
v_{t}=\frac{\mathrm{E}_{t}\left[J_{t+1}^{\prime} v_{t+1}\right]}{\mathrm{E}_{t}\left[J_{t+1}^{\prime}\right]}, \quad t=0,1,
$$

where $J_{t+1}^{\prime}$ is a shorthand for $J_{t+1}^{\prime}\left(W_{t+1}\right), J_{2}=-\exp \left\{-\alpha W_{2}\right\}$, and $v_{2}=\left(D-P_{1}\right)^{2}$. We then have the following result: 
Proposition 2 The equilibrium price of variance swap at dates 0 and 1 are given by:

$$
\begin{aligned}
v_{0} & =(1-\delta)\left(\lambda_{0}+\lambda\right)+(1-\delta) \frac{\frac{1}{2} \alpha^{2} \lambda^{2}}{1-\frac{1}{2} \alpha^{2} \lambda}, \quad v_{1}=(1-\delta) \sigma^{2}, \\
\mathrm{E}\left[v_{1}-v_{0}\right] & =-(1-\delta) \frac{\frac{1}{2} \alpha^{2} \lambda^{2}}{1-\frac{1}{2} \alpha^{2} \lambda}<0 .
\end{aligned}
$$

Thus, from (17b), the change in the price of variance swap provides a measure of $\mathrm{V}\left[\sigma^{2}\right]=\lambda^{2}$. Since the price of the variance swap can be viewed as equivalent to VIX squared, this proposition then predicts that VIX increases when uncertainty rises ahead of an announcement, and it decreases when the uncertainty is resolved. In what follows, we will use changes in VIX to gauge changes in uncertainty.

\subsection{Return and Variance}

We now examine the returns on the stock over the two periods, from 0 to 1 and 1 to 2 . Define the stock returns as follows:

$$
R_{t}=P_{t}-P_{t-1}, \quad t=1,2
$$

From the stock prices given in Proposition 1, we have the following results for the mean and variance of returns on the stock over the two periods:

$$
\begin{aligned}
& \mathrm{E}\left[R_{1}\right]=\alpha \delta\left(\lambda_{0}+\lambda\right)+\frac{\frac{1}{2} \alpha^{3} \lambda^{2}}{1-\frac{1}{2} \alpha^{2} \lambda}>0, \\
& \mathrm{~V}\left[R_{1}\right]=\delta\left(\lambda_{0}+\lambda\right)+\alpha^{2}(1-\delta)^{2} \lambda^{2} .
\end{aligned}
$$

and

$$
\begin{aligned}
\mathrm{E}\left[R_{2}\right] & =\alpha(1-\delta)\left(\lambda_{0}+\lambda\right)>0, \\
\mathrm{~V}\left[R_{2}\right] & =(1-\delta)\left(\lambda_{0}+\lambda\right)+\alpha^{2}(1-\delta)^{2} \lambda^{2} .
\end{aligned}
$$

First, we observe that the expected return over both periods are positive, compensating the risks over each period. But, they are compensating for different risks. For the second period, the expected return, $\mathrm{E}\left[R_{1}\right]$ in $(20)$, is proportional to $(1-\delta)\left(\lambda_{0}+\lambda\right)$, which is the risk associated with the remaining part of the news revealed at the announcement (i.e., $\varepsilon_{2}$ ). It has nothing to do with the uncertainty about $\sigma^{2}$, which is measured by $\lambda^{2}$. For the first period, however, the expected return, $\mathrm{E}\left[R_{1}\right]$ given in (19), compensates for both the news risk and the uncertainty. In particular, the first term reflects the risk premium for the news 
risk, which is proportional to the fraction of news revealed in this period $\delta$, times its expected impact $\left(\lambda_{0}+\lambda\right)$. The second term, however, reflects the risk premium for uncertainty.

Using return variance as a measure of risk, we also see that the variances of $R_{1}$ and $R_{2}$ capture different combinations of the two risks. The first term in both $\mathrm{V}\left[R_{1}\right]$ and $\mathrm{V}\left[R_{2}\right]$ arises from the news risk, both proportional to $\lambda_{0}+\lambda$, one with a weight of $\delta$ while the other with a weight of $1-\delta$. The second term, however, comes from the impact risk or uncertainty - its resolution attributes to the variability in the stock price at $t=1, P_{1}$.

The returns over the two periods, as shown by their means and variances, can exhibit a rich set of patterns. In order to further explore their underlying structure, we now consider their ratio, the return-variance ratio (RVR), as a measure of risk premium or the price of risk:

$$
R V R_{t}=\frac{\mathrm{E}\left[R_{t}\right]}{\mathrm{V}\left[R_{t}\right]}, \quad t=1,2 .
$$

We use the return-variance ratio instead of the Sharpe ratio (the return-volatility ratio) to measure risk premium mainly because the former is scalable over time while the latter is not. Given that in the empirical analysis, the time window may vary when measuring returns, the return-variance ratio is a more appropriate measure to compare the risk premiums for different time windows and different risks.

\section{Proposition 3 Let}

$$
\lambda^{*}\left(\lambda_{0}\right)=\frac{\sqrt{2}}{\alpha^{2}}+\frac{(2-\sqrt{2}) \lambda_{0}}{4+\alpha^{2} \lambda_{0}} \in\left[\sqrt{2} / \alpha^{2}, 2 / \alpha^{2}\right) .
$$

When $\lambda>\lambda^{*}\left(\lambda_{0}\right)$, we have:

$$
\begin{aligned}
& \mathrm{E}\left[R_{1}\right]>\mathrm{E}\left[R_{2}\right], \\
& \frac{\mathrm{E}\left[R_{1}\right]}{\mathrm{V}\left[R_{1}\right]}>\frac{\mathrm{E}\left[R_{1}\right]}{\mathrm{V}\left[R_{1}\right]} .
\end{aligned}
$$

An immediate implication of Proposition 3 is that if $\lambda>\lambda^{*}\left(\lambda_{0}\right)$, that is, if uncertainty is sufficiently high, then the expected return and return-to-variance ratio for the first period are both higher than their counterparts for the second period.

\subsection{A Special Case with Leakage of News but No Uncertainty}

To better illustrate our model's implications, it is instructive to consider the special case with leaks but no uncertainty. This corresponds to $\sigma^{2}=\lambda_{0}, \lambda=0$, and $0 \leq \delta \leq 1$. From 
(19) and (20), we immediately have:

$$
\begin{aligned}
& \mathrm{E}\left[R_{1}\right]=\alpha \delta \sigma^{2}, \quad \mathrm{~V}\left[R_{1}\right]=\delta \sigma^{2}, \\
& \mathrm{E}\left[R_{2}\right]=\alpha(1-\delta) \sigma^{2}, \quad \mathrm{~V}\left[R_{2}\right]=(1-\delta) \sigma^{2} .
\end{aligned}
$$

At this point, it is apparent that the return-to-variance ratio for the two periods are identical, both equal to $\alpha$.

Corollary 1 If the variance $\sigma^{2}$ is known ex ante, i.e., $\lambda=0$, for any $0 \leq \delta \leq 1$, the return-to-variance ratios in the two dates are identical:

$$
\frac{\mathrm{E}\left[R_{1}\right]}{\mathrm{V}\left[R_{1}\right]}=\frac{\mathrm{E}\left[R_{2}\right]}{\mathrm{V}\left[R_{2}\right]}=\alpha
$$

Here, for $\delta$ equal to 0 or 1, the ratio is given by the limit as $\delta$ converges to 0 or 1 , respectively.

The key takeaway from this special case is that if the leakage prior to its actual announcement is about the content of the announcement, then the return-to-variance ratios before and after the announcement have to be identical. Although we derive this result from a simple model, the intuition should be more general: the same source of risk should lead to the same return-to-variance ratios.

\subsection{Empirical Implications}

Our model leads to clear predictions about the market behavior surrounding macroeconomic announcements. First, it identifies three relevant time periods.

- The first period is the "accumulation period", which corresponds to the time prior to date 0 . Although this period is not explicitly specified in the model, it is clear that, if $\lambda$ itself is a random variable, it would be realized during the time up to date 0 . A large realization of $\lambda$ by date 0 is what we refer to as heightened uncertainty.

- The second period is the "pre-announcement period", which is from date 0 to 1 in the model. During this period, the uncertainty about the news' impact $\sigma$ is resolved. In addition, there can be partial leakage of the news itself, as measured by $\delta$.

- The third period is the "post-announcement period", which is from date 1 to 2 in the model. During this period, the news is released and its impact on asset payoff is realized.

Next, the model yields the following predictions on the returns, return-to-variance ratios and changes in VIX over these three periods. 
Prediction 1 Positive stock returns over the pre-announcement period occur whenever there is heightened uncertainty which is resolved prior to macroeconomic announcements.

In particular, pre-announcement return is not unique to FOMC and can also arise for other important macroeconomic announcements. Depending on the level of heightened uncertainty, the pre-announcement return can vary over different announcements, both cross different types of announcements and within each type of announcements.

Prediction 2 Prior to a pre-scheduled macroeconomic announcement (including FOMC), if there were only leakage about the content of announcement itself but no uncertainty concerning its impact, the return-to-variance ratios for the pre- and post-announcement periods should be identical, i.e., $\frac{\mathrm{E}\left[R_{1}\right]}{\mathrm{V}\left[R_{1}\right]}=\frac{\mathrm{E}\left[R_{2}\right]}{\mathrm{V}\left[R_{2}\right]}$.

A rejection of Prediction 2, i.e., evidence that the two return-to-variance ratios differ, would suggest that a second risk is present, independent from news leakage.

Prediction 3 The drop in VIX is a proxy of heightened uncertainty $\lambda$. A larger preannouncement VIX drop leads to a higher return in the pre-announcement period.

In addition, when uncertainty heightens substantially during the days prior the announcement, i.e., the accumulation period, it should lead to a rise in VIX accompanied by a price drop, followed by a higher return and variance before and after the announcement.

Prediction $4 A$ VIX build-up in the accumulation period captures an increase in uncertainty $\lambda$, which leads to a contemporaneous drop in price, a higher return and larger VIX drop in the pre-announcement period.

Finally, given that a rise in VIX is a proxy for heightened uncertainty before scheduled announcements, it is plausible that an unexpected increase in VIX reflects an unanticipated heightened uncertainty, with similar predictions about subsequent returns and VIX changes.

Prediction 5 Unanticipated spikes in VIX will be followed by VIX reversals and high returns.

In what follows, we will test these predictions using macroeconomic announcements, as well as VIX spikes, capturing both anticipated and unanticipated arrival of heightened uncertainty. 


\section{Data}

We use several data sets in our analysis. For macroeconomic announcements, we consider the releases of major U.S. macroeconomic indicators. We include the top six macroeconomic indicators ranked by Bloomberg's "relevance score" (as of 2019): total Nonfarm Payroll employment (NFP), initial claims for unemployment insurance (INC), Gross Domestic Production (GDP), consumer price index (CPI), the Institute for Supply Management's manufacturing index (ISM), and the preliminary release of the Consumer Sentiment Index (CSI). We also include industrial production (IP), personal income (PI), housing starts (HST), and producer price index (PPI), partly because they have been used in previous studies. All of these macroeconomic indicators are released monthly (including GDP, which has one preliminary announcement and two revisions for each quarter). Data on these announcements are from the websites of their releasing entities.

The nature and timing of these announcements vary substantially. We describe them below in detail. Except for ISM and CSI, all other economic indicators are public indices released by government agencies at either 8:30 am or 9:15 am (only for IP), Eastern Time. ISM an CSI are economic indicators released by private institutions. ISM is released at 10:00 am, while CSI's release time varies from 9:35 am to 10:00 am during our sample period. We exclude macroeconomic announcement days that coincide with FOMC announcement days to avoid potential confounding effect.

We also include the FOMC announcement, which occurs eight times a year. The timing of the FOMC announcements are based on the time-stamp of Bloomberg and Dow Jones news wires. We follow the same method of Lucca and Moench (2015) and Fleming and Piazzesi (2005), and extend the sample period to May 2018. We focus most of our analysis on the sample from September 1994 to May 2018. During this period, there are in total 190 scheduled releases of FOMC statements. From September 1994 to March 2011, 131 releases are consistently made within a few minutes around 2:15 pm, with only one exception, March 26, 1996, on which the release time was pre-announced to be in the morning because of the Chairman's other duties. From April 2011 to January 2013, seven releases are around 2:15 pm and eight releases are around 12:30 pm, one hour and forty five minutes earlier to accommodate the Chairman's press briefings at 2:15 pm. From February 2013 to May 2018, all of the 43 FOMC releases are around 2:00 pm. For the period before 1994, there is no official announcement and market participants need to inferred policy decisions through the Fed's open market operations, usually on the day after the FOMC meeting. 
For all announcements, we define the pre-announcement period as the window from the close of the previous trading day $(4 \mathrm{pm})$ to five minutes prior to the exact release time (ann-5min). Using the market close as a natural starting point, our construction of the pre-announcement window is consistent with the one often used for FOMC and allows for a unified comparison of the pre-announcement drift across different releases. Similarly, we define the post-announcement period as the window from five minutes prior to the release to the close of the announcement day.

We rely on the S\&P 500 index futures, which are traded almost around the clock, to calculate market returns around announcements. We obtain the transaction-level data on E-mini S\&P 500 index futures from September 1997 to May 2018 from the Chicago Mercantile Exchange (CME). Prior to September 1997, when the E-mini contract was not available, we use transaction-level data on the standard ("big") S\&P 500 index futures from the CME. ${ }^{12}$ To calculate market returns over a given time period $\left[t_{1}, t_{2}\right]$, we first pick the most active S\&P 500 index futures contract as the one with the highest trading volume on the trading day of $t_{2}$, and then calculate the return as the percentage change of the last transaction price of this futures contract before time $t_{2}$, relative to the last transaction price of the same contract before time $t_{1}{ }^{13}$

To calculate the changes in VIX around announcements, we rely on the intraday VIX tick data and the transaction-level data on VIX futures obtained from the Chicago Board Options Exchange (CBOE). For announcements released within the regular trading hours (FOMC, ISM and CSI), we calculate the changes in VIX around announcements based on the intraday VIX tick data. ${ }^{14}$ For other announcements whose releases fall outside the regular trading hours, we calculate the change in VIX based on VIX futures, using method similar to how we calculate returns from S\&P 500 index futures prices. The VIX futures data starts to cover the non-regular trading hours only after December 2010. For this reason, the

\footnotetext{
${ }^{12}$ Due to data limit, prices of standard S\&P 500 futures contracts are not available at non-regular trading hours. Therefore, the pre-announcement returns for macro-announcements that are released before market opens are only available after September 9, 1997, when E-mini S\&P 500 index futures started trading. We have missing futures trading data on eight trading days in our sample period. One of these eight trading days, January 29, 2014, is a scheduled FOMC release day. For these eight trading days, we rely on the transaction level S\&P 500 index data obtained from TAQ to construct the market return.

${ }^{13}$ We choose the most active futures contract as the one with the highest volume, which is usually the nearest-term contract and occasionally the next contract during rolling forward weeks.

${ }^{14}$ The intraday VIX tick data contains only VIX values within the regular trading hours for most of our sample period. The daily coverage starts from 10:00 am since January 2, 1992. The coverage is then extended to 9:30 am since January 2, 2004, and to 3:15 am since April 15, 2016. The intraday VIX data ends at 4:00 pm before January 2, 2004 and at 4:15 pm afterward.
} 
number of events that we can calculate VIX changes around announcements are fewer for those released outside of the regular trading hours.

\section{Empirical Results: Macroeconomic Announcements}

\subsection{Pre-Announcement Returns}

As outlined by Prediction 1 of our model, positive pre-announcement returns arise from the resolution of heightened impact uncertainty prior to the scheduled news announcement. As such, we should be able to find significant pre-announcement returns not only for FOMC, but also for other macro announcements, as long as they bring substantial heightened uncertainty. To test this prediction, we investigate the pre-announcement returns for a set of well known macroeconomic announcements. Given the extensive coverage on the pre-FOMC return in the existing literature, we focus our discussion on the positive pre-announcement returns for non-FOMC announcements, which have not been documented prior to our paper.

The pre-announcement returns are reported in Table 1, using data from September 1994 through May 2018. To achieve a consistent comparison across different announcements as well as to capture the price movement immediately before the announcement, we define the the pre-announcement window as from the previous day's close at 4 pm to 5 minutes before the scheduled releases. Consistent with Prediction 1, we find that pre-announcement returns are significant for NFP, ISM, and GDP - three macro events that are known to be important and highly ranked by Bloomberg's relevance score. Specifically, the average preannouncement return is 10.10 basis points for NFP with a t-stat of 3.63; 9.14 basis points for ISM with a t-stat of 2.10; 7.46 basis points for GDP with a t-stat of 2.08. These results are not driven by outliers. After removing the $1 \%$ top and bottom returns, the average pre-announcement drift remains important and significant: 9.80 basis points for NPF, 10.31 basis points for ISM, and 6.09 basis points for GDP.

Benchmarked against the average market return of 3.61 basis points per day for the same sample period, the economic magnitudes of these pre-announcement returns of 7 to 10 basis points are rather significant. Further excluding announcement days, the benchmark return shrinks to less than one basis points per non-announcement day, making the contrast even more stark. Moreover, these pre-announcement returns are not full-day returns - the preannouncement returns for NFP and GDP are realized overnight, from the previous day's close at 4pm to 8:25 am, while those for ISM are from the previous day's close to 9:55 am. The comparable benchmark return should therefore be measured from close to open, which, 


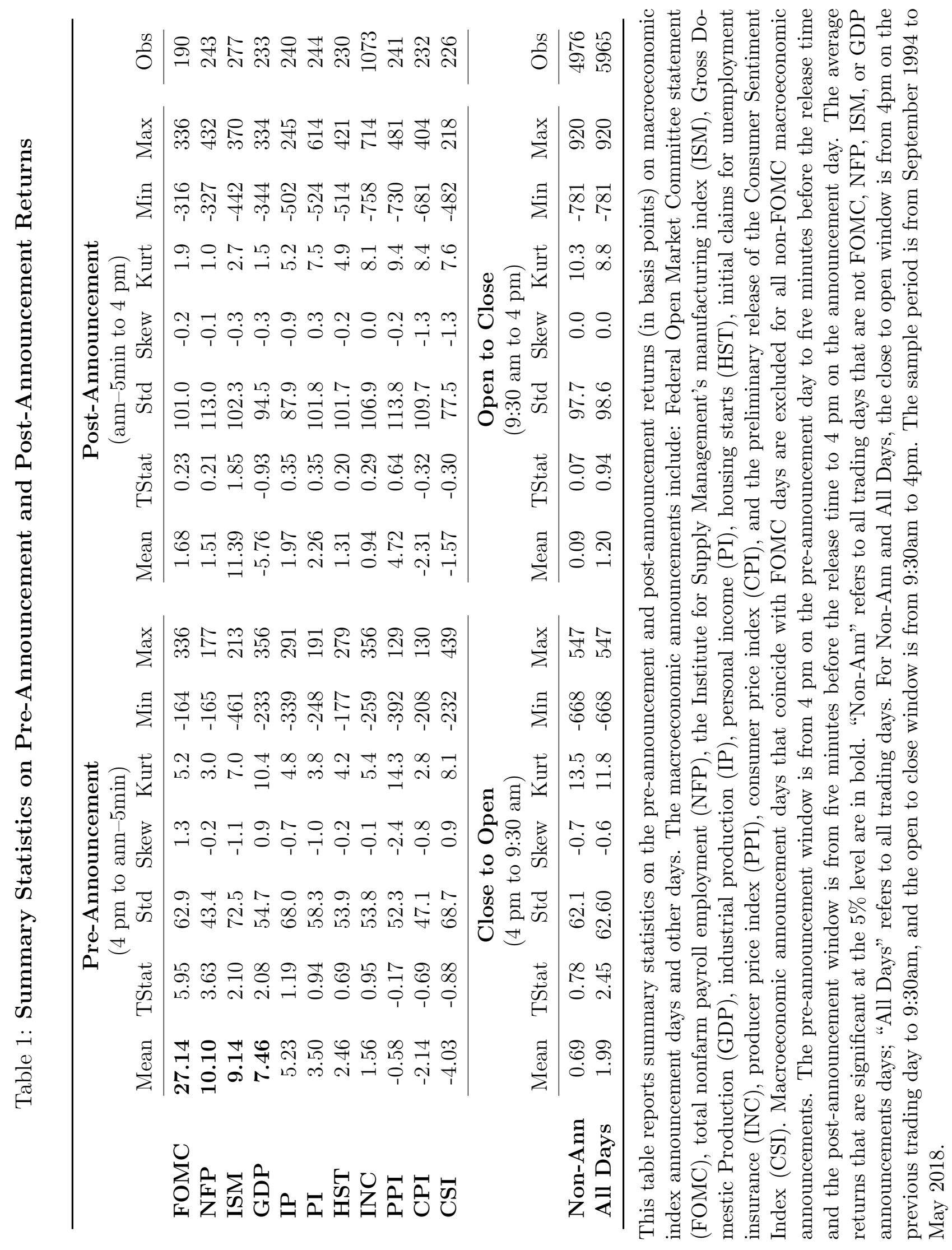


as shown in Table 1, is 1.99 basis points when averaged across all trading days and 0.69 basis points averaged across non-announcement days.

One important observation emerging from the results in Table 1 is that there is a substantial heterogeneity across macro announcements. In the context of our model, the varying levels of pre-announcement return is a reflection of the varying magnitudes of impact uncertainty. Indeed, the variation in impact uncertainty and its asset-pricing implications can be examined not only across different macro indicators, as listed in Table 1, but also across different announcement days for the same macro indicator, which will be examined later in the section.

Focusing on the cross-indicator variation in Table 1, we see that, not surprisingly, the preannouncement returns associated with FOMC, with an average value of 27.14 basis points per event, are found to be the largest, indicating strong heightened uncertainty brought upon by the impending FOMC announcements, consistent with the abundant anecdotes of investors anxiously awaiting the FOMC outcome. ${ }^{15}$ On the other hand, some of the macroeconomic indicators are found to have insignificant pre-announcement returns, indicating their low impact uncertainty. Indeed, lining up the macro announcements by their pre-announcement returns, the ranking is consistent with the relative importance of the announcements, both intuitively perceived by market participants and empirically documented by prior literature. ${ }^{16}$ From this perspective, our results add discipline as well as richness to the literature that focuses mostly on the pre-FOMC drift. More importantly, we add a theoretical foundation to the unusual risk-and-return tradeoff surrounding the announcements that has puzzled the literature on pre-FOMC drift.

To further illustrate the return patterns before the releases of the non-FOMC macroeconomic indicators, Figure 1 plots the average cumulative return of the E-mini S\&P500 index futures, minute by minute, during the pre-announcement window. ${ }^{17}$ For NFP, ISM, and GDP, there is a clear upward drift of the E-mini S\&P500 index futures prices hours ahead of

\footnotetext{
${ }^{15}$ Our calculation of the pre-announcement returns for FOMC is lower than those reported in Lucca and Moench (2015) for two reasons. First, our pre-announcement window starts from $4 \mathrm{pm}$ on the previous day, shorter than the 24-hours window used by Lucca and Moench (2015), excluding the small price run-up from $2 \mathrm{pm}$ to $4 \mathrm{pm}$ on the day prior to the announcement. Second, we updated the sample period of Lucca and Moench (2015) to include the post-2011 pre-FOMC returns, which are on average smaller because of the unusual monetary policy post 2008 .

${ }^{16}$ For example, among the 36 macroeconomic announcements analyzed by Gilbert, Kurov, and Wolfe (2018), nonfarm payrolls has the largest explanatory power for U.S. Treasury yields, followed by ISM.

${ }^{17}$ We skip the 15 -minute trading halt from $4: 15 \mathrm{pm}$ to $4: 30 \mathrm{pm}$ and the daily maintenance hour from 5:00pm to 6:00pm for E-mini S\&P500 futures.
} 


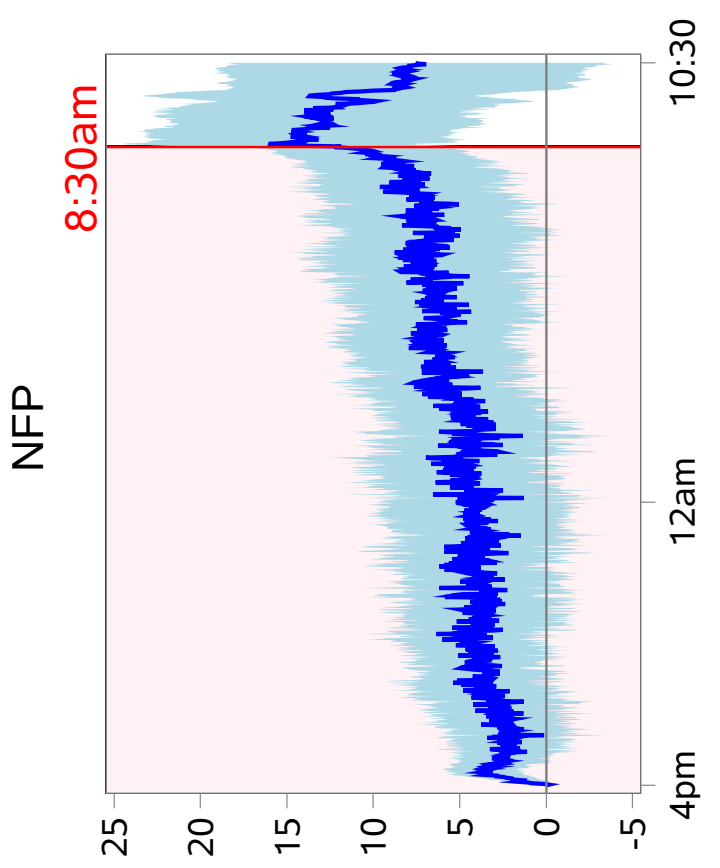

(sdq) łəУ әм!̣eןnunว

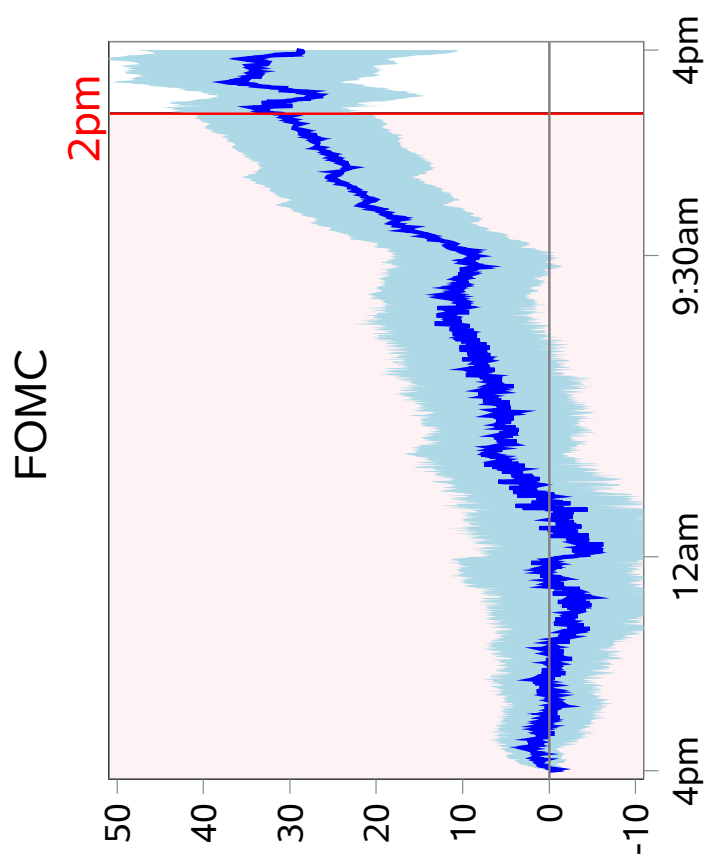

(sdq) łəу 'unכ
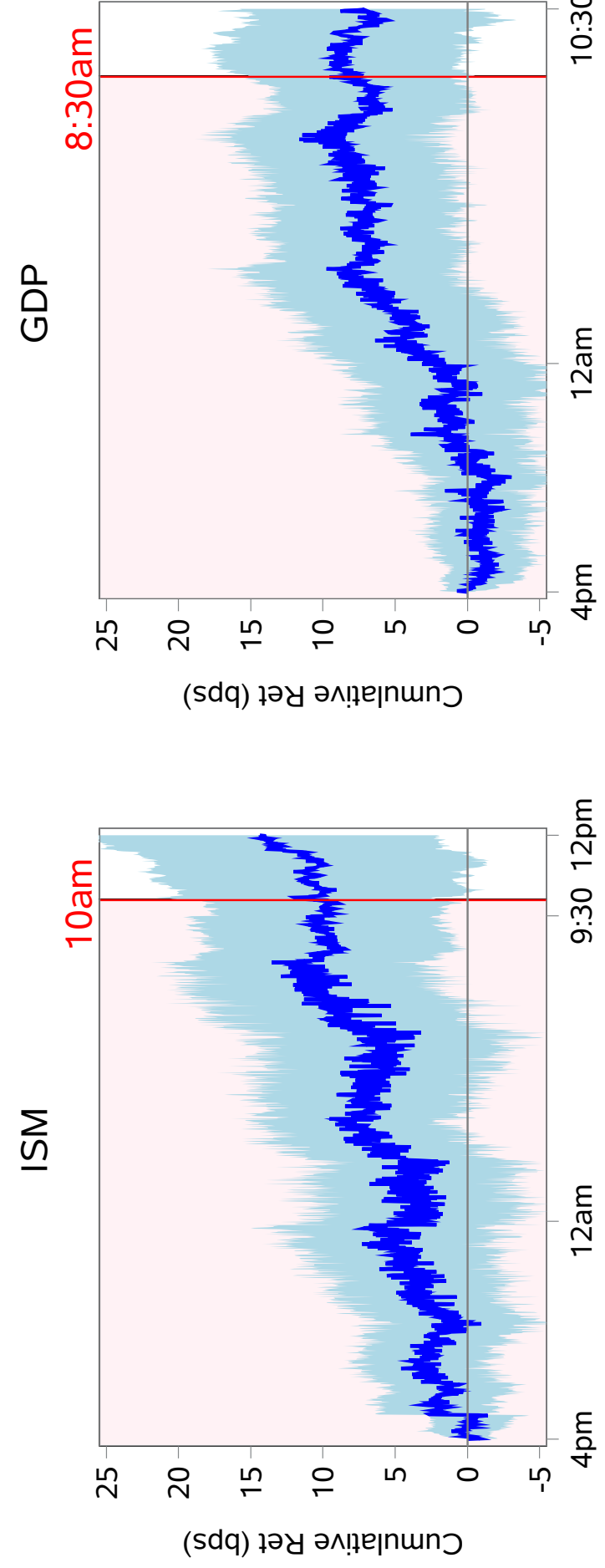

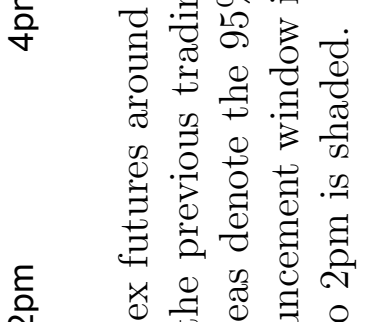

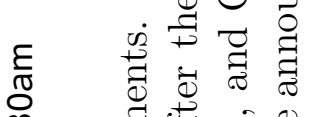

हृष्ठ

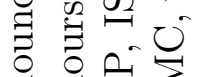

寻宁空

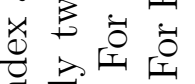

$\exists$ 吾 $\dot{0}$

.

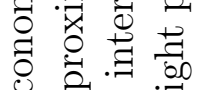

仓

०

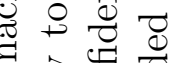

घ

요.

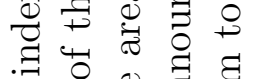

\&

เि 위를

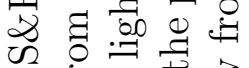

สี

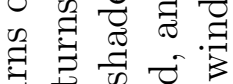

$\exists$ 苟

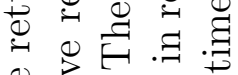

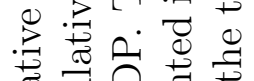

票官帚

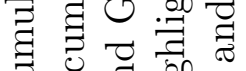

उ. ซี

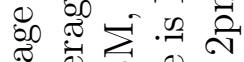

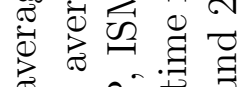

¿ 0 م

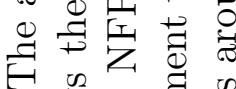

E क

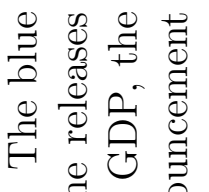

$\ddot{-} \circlearrowright 00$

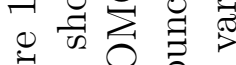

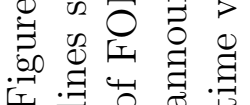


their releases. As the releases are made early in the morning, with NFP and GDP at 8:30 am and ISM at $10 \mathrm{am}$, most of the pre-announcement returns are in fact earned outside of the regular trading hours, making the overnight return uniquely important. Indeed, skipping the overnight window is the reason why earlier studies missed this important result. This is also the reason why the pre-FOMC return was captured first in the literature, since, as shown in Figure 1, a significant portion of the pre-FOMC return is realized during the regular trading hours.

Examining the robustness of our pre-announcement results, Table 2 reports the preannouncement returns over the three subperiods of 1994-2000, 2001-2011, and 2012-2018. Grouping all four macro announcement together, the average pre-announcement returns per event vary from 7 to 16 basis points over the three subperiods, and are all statistically significant. Separating the macro announcements into FOMC and non-FOMC (NFP, ISM, and GDP), the sub-period performance for FOMC remains large and significant pre-2011 and becomes insignificant during 2012-2018. By contrast, the performance of the non-FOMC macro announcements remains stable and significant across all three subperiods. In particular, during the last subperiod of 2012-2018, the pre-announcement return is on average 6.98 basis point and statistically significant for the non-FOMC macro announcements, compared with the statistically insignificant 3.96 basis points for the FOMC announcements.

Another way to gauge and compare the magnitudes of the pre-announcement return is by measuring the returns annually - adding the pre-announcement returns across all events within a year. As shown in Panel B of Table 2, the pre-announcement returns, realized over 44 macro announcements per day, add up to $5.66 \%$ per year, a significant fraction of the total stock return of $9.10 \%$. Separating the four macro announcements into FOMC and non-FOMC (i.e., NFP, IMS and GDP), the pre-announcement returns add up to an annual number of $3.41 \%$ for non-FOMC, and $2.17 \%$ for FOMC. From this yearly perspective, we see that, while FOMC is in general more intense than other macro announcements, its cumulative impact is in fact smaller when compared to the other three macro announcements combined. Their relative importance also varies across different subperiods along with the changing macroeconomic conditions. For example, post 2011, the relative contribution of FOMC diminishes to $0.32 \%$ per year while the contribution of the other three macro indicators remains stable at $2.51 \%$ per year. Also interesting is the fact that, while the market return performs poorly at $1.97 \%$ per year during the subperiod of 2000-2011, the pre-announcement returns remain large at $6.54 \%$ per year. 
Table 2: Pre-Announcement Returns

\begin{tabular}{|c|c|c|c|c|}
\hline \multirow[t]{3}{*}{ Panel } & \multicolumn{4}{|c|}{ A: Pre-announcement return per event (in basis points) } \\
\hline & \multicolumn{3}{|c|}{ Macro Announcements } & \multirow{2}{*}{$\begin{array}{c}\text { All Days } \\
\text { (close-to-close) }\end{array}$} \\
\hline & All 4 & Ex FOMC & FOMC & \\
\hline $1994-2000$ & $\begin{array}{l}\mathbf{1 6 . 0 0} \\
{[4.22]}\end{array}$ & $\begin{array}{c}\mathbf{9 . 6 0} \\
{[2.17]}\end{array}$ & $\begin{array}{l}35.81 \\
{[5.55]}\end{array}$ & $\begin{array}{l}\mathbf{6 . 9 5} \\
{[2.62]}\end{array}$ \\
\hline $2001-2011$ & $\begin{array}{l}\mathbf{1 4 . 8 7} \\
{[4.58]}\end{array}$ & $\begin{array}{l}\mathbf{1 0 . 8 8} \\
{[3.16]}\end{array}$ & $\begin{array}{l}35.55 \\
{[4.27]}\end{array}$ & $\begin{array}{c}\mathbf{0 . 7 8} \\
{[0.30]}\end{array}$ \\
\hline 2012-2018 & $\begin{array}{c}7.02 \\
{[2.54]}\end{array}$ & $\begin{array}{c}\mathbf{6 . 9 8} \\
{[2.27]}\end{array}$ & $\begin{array}{c}3.96 \\
{[0.74]}\end{array}$ & $\begin{array}{l}\mathbf{5 . 1 4} \\
{[2.62]}\end{array}$ \\
\hline 1994-2018 & $\begin{array}{l}\mathbf{1 2 . 8 6} \\
{[6.49]}\end{array}$ & $\begin{array}{c}\mathbf{9 . 4 8} \\
{[4.40]}\end{array}$ & $\begin{array}{c}\mathbf{2 7 . 1 4} \\
{[5.95]}\end{array}$ & $\begin{array}{c}\mathbf{3 . 6 1} \\
{[2.39]}\end{array}$ \\
\hline \multicolumn{5}{|c|}{ Panel B: Pre-announcement return per year (in percent) } \\
\hline & \multicolumn{3}{|c|}{ Macro Announcements } & All Days \\
\hline & All 4 & Ex FOMC & FOMC & (close-to-close) \\
\hline $1994-2000$ & 7.04 & 3.46 & 2.86 & 17.51 \\
\hline $2001-2011$ & 6.54 & 3.92 & 2.84 & 1.97 \\
\hline 2012-2018 & 3.09 & 2.51 & 0.32 & 12.95 \\
\hline 1994-2018 & 5.66 & 3.41 & 2.17 & 9.10 \\
\hline \# events/yr & 44 & 36 & 8 & 252 \\
\hline
\end{tabular}

This table reports the average and the annualized pre-announcement returns on important macroeconomic releases, for the full sample as well as the three subperiods. "All 4" includes FOMC, NFP, ISM, and GDP, and "Ex FOMC" excludes FOMC from the four. In Panel A, the average pre-announcement returns across events and the associated t-statistics (in square brackets) are reported. The average returns that are significant at the $5 \%$ level are in bold. In Panel B, the annualized pre-announcement returns are calculated by multiplying the average pre-announcement returns with the number of macroeconomic releases per year. "All Days" refers to all trading days in the sample period; and the daily close-to-close returns on S\&P 500 index are used to calculate the respective average and annualized returns.

The same message is conveyed, at a higher frequency, by Figure 2, which plots the yearly pre-announcement returns for the four macroeconomic announcements combined (red squares), FOMC only (green crosses), and the three non-FOMC combined (blue circles). As a comparison, the annual market returns are also plotted in the background (gray diamonds). One striking feature of this plot is that, while the overall market experiences some rather negative returns throughout the sample period, rarely do the yearly pre-announcement returns 


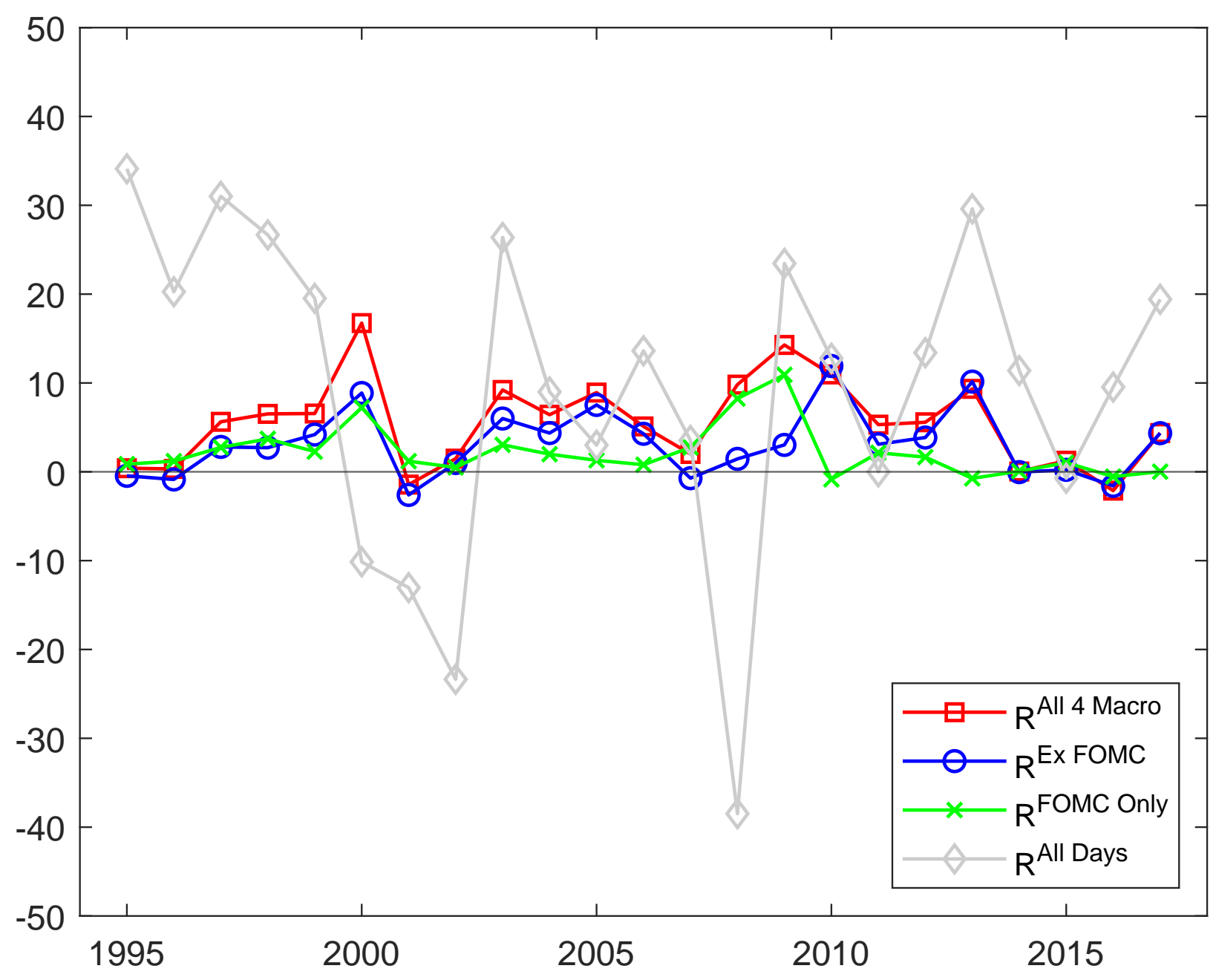

Figure 2: Time-series of yearly S\&P 500 index returns realized pre-announcement on event days. For macroeconomic index announcements, the pre-announcement returns realized within each calendar year are used to calculate the respective yearly return. "All 4 Macro" includes FOMC, NFP, ISM, and GDP, and "Ex FOMC" excludes FOMC from the four. "All Days" refers to all trading days and the daily close-to-close returns on S\&P 500 are used to calculate the respective yearly return.

dip significantly below zero. Another interesting feature is that pre-2010, there is quite a bit of similarity between FOMC and non-FOMC in terms of their time-series variation. Post 2010, however, the yearly pre-announcement returns of FOMC flatten out while those for non-FOMC remain relatively robust. This, of course, is likely related to the unconventional monetary policy after the 2008-09 financial crisis. 


\subsection{Return-to-Variance Ratios}

As outlined by Prediction 2 of our model, in presence of only one risk (i.e., the news risk), the return-to-variance ratio during the pre-announcement period should be identical to that of the post-announcement period, regardless of information leakage. As a direct test of the one-risk hypothesis, we compare the return-to-variance ratios, which are invariant over time scale, for the two periods immediately before and after the announcement. The preannouncement window is as defined earlier, while the post-announcement window is from the end of the pre-announcement window to the close of the announcement day at $4 \mathrm{pm}$.

As reported in Table 3, the return-to-variance ratio is substantially higher pre- than post-announcement. With the four macroeconomic announcements combined, the preannouncement returns have an average of 12.86 basis points and variance of 0.36 basis points, yielding a return-to-variance ratio of 35.53 with a highly significant t-stat of 5.27. The postannouncement returns, by comparison, are substantially smaller in magnitudes (2.50 basis points) and higher in variance (1.07 basis points), yielding a return-to-variance ratio of a mere 2.34 and is statistically insignificantly from zero. The difference of the return-to-variance ratios is 33.19 and statistically significant at the $1 \%$ level, rejecting the hypothesis that the pre- and post-announcement returns have the same return-to-variance ratio. The patterns are similar for FOMC as well as for the three non-FOMC macroeconomic indicators. Table 3 further examines and documents the robustness of this result over the three subperiods of 1994-2000, 2001-2011, and 2012-2018.

Overall, the apparent discrepancy in return-to-variance ratio during the pre-and postannouncement periods strongly indicates the presence of at least one more risk surrounding the macroeconomic announcements and is counter to the hypothesis that the large preannouncement return is driven only by information leakage about the news risk alone.

\subsection{Resolution of Impact Uncertainty}

According to Prediction 3 of the model, the pre-announcement reduction in VIX, reflecting the resolution of impact uncertainty, can be used as a proxy for the magnitude of impact uncertainty $\lambda$, which, in turn, has a positive impact on the pre-announcement returns.

Exploring the cross-announcement variation in the magnitude of impact uncertainty $\lambda$, we sort macroeconomic announcement days into a high-uncertainty group, defined as the top $30 \%$ of the announcements with the largest reduction in VIX during the pre-announcement 
Table 3: Pre- and Post-Announcement Return-to-Variance Ratios

\begin{tabular}{|c|c|c|c|c|c|c|c|}
\hline & \multicolumn{3}{|c|}{ Pre } & \multicolumn{3}{|c|}{ Post } & \multirow{2}{*}{$\frac{\text { Pre-Post }}{\text { Ret/Var }}$} \\
\hline & $\begin{array}{l}\text { Ret } \\
\text { (bps) }\end{array}$ & $\begin{array}{l}\text { Var } \\
(\mathrm{bps})\end{array}$ & Ret/Var & $\begin{array}{l}\text { Ret } \\
(\mathrm{bps})\end{array}$ & $\begin{array}{l}\text { Var } \\
\text { (bps) }\end{array}$ & Ret/Var & \\
\hline All 4 Macro & $\begin{array}{l}\mathbf{1 2 . 8 6} \\
{[6.49]}\end{array}$ & 0.36 & $\begin{array}{l}35.53 \\
{[5.27]}\end{array}$ & $\begin{array}{c}2.50 \\
{[0.73]}\end{array}$ & 1.07 & $\begin{array}{c}2.34 \\
{[0.73]}\end{array}$ & $\begin{array}{l}33.19 \\
{[4.45]}\end{array}$ \\
\hline Ex FOMC & $\begin{array}{c}\mathbf{9 . 1 5} \\
{[4.20]}\end{array}$ & 0.35 & $\begin{array}{l}\mathbf{2 6 . 3 8} \\
{[3.49]}\end{array}$ & $\begin{array}{c}2.71 \\
{[0.70]}\end{array}$ & 1.08 & $\begin{array}{c}2.50 \\
{[0.70]}\end{array}$ & $\begin{array}{l}23.87 \\
{[2.86]}\end{array}$ \\
\hline FOMC Only & $\begin{array}{l}\mathbf{2 7 . 1 4} \\
{[5.95]}\end{array}$ & 0.40 & $\begin{array}{l}68.58 \\
{[5.41]}\end{array}$ & $\begin{array}{c}1.68 \\
{[0.23]}\end{array}$ & 1.02 & $\begin{array}{c}1.64 \\
{[0.23]}\end{array}$ & $\begin{array}{l}\mathbf{6 6 . 9 3} \\
{[4.59]}\end{array}$ \\
\hline \multicolumn{8}{|c|}{ Subperiods for All 4 Macro } \\
\hline $1994-2000$ & $\begin{array}{l}\mathbf{1 6 . 0 0} \\
{[4.22]}\end{array}$ & 0.28 & $\begin{array}{l}\mathbf{5 6 . 7 3} \\
{[3.71]}\end{array}$ & $\begin{array}{l}12.47 \\
{[1.57]}\end{array}$ & 1.23 & $\begin{array}{l}10.11 \\
{[1.51]}\end{array}$ & $\begin{array}{l}46.62 \\
{[2.79]}\end{array}$ \\
\hline $2001-2010$ & $\begin{array}{l}\mathbf{1 5 . 2 2} \\
{[4.54]}\end{array}$ & 0.47 & $\begin{array}{l}32.27 \\
{[3.52]}\end{array}$ & $\begin{array}{c}-3.14 \\
{[-0.55]}\end{array}$ & 1.34 & $\begin{array}{l}-2.33 \\
{[-0.56]}\end{array}$ & $\begin{array}{l}\mathbf{3 4 . 6 0} \\
{[3.43]}\end{array}$ \\
\hline 2011-2018 & $\begin{array}{c}7.63 \\
{[2.62]}\end{array}$ & 0.26 & $\begin{array}{c}\mathbf{2 9 . 2 2} \\
{[2.46]}\end{array}$ & $\begin{array}{c}3.83 \\
{[0.88]}\end{array}$ & 0.59 & $\begin{array}{c}6.52 \\
{[0.87]}\end{array}$ & $\begin{array}{l}22.70 \\
{[1.61]}\end{array}$ \\
\hline
\end{tabular}

"All 4 Macro" includes FOMC, NFP, ISM, and GDP. "Ex FOMC" excludes FOMC from the four. Post-announcement begins 5 minutes before the announcement and ends at the announcement day's close at $4 \mathrm{pm}$. Pre-announcement begins at the previous day's close and ends at the beginning of the post-announcement window. Numbers that are significant at the $5 \%$ level are in bold. The sample period is from September 1994 to May 2018.

period, and a low-uncertainty group, defined as the remaining announcements. ${ }^{18}$ Panel A of Table 4 reports $\Delta$ VIX and return for both the pre-announcement and post-announcement periods. As predicted by the model, the high uncertainty announcements (larger $\lambda$ ), on average have much larger pre-announcement returns. Using all four macroeconomic indicators, we observe that in the high uncertainty group of events, the average pre-announcement return is positive at 68.23 basis points and highly statistically significant, whereas in the low uncertainty group, the average pre-announcement return is negative at -6.96 basis points. The average pre-announcement return difference between the high- and low-uncertainty group is 75.19 basis points and statistically significant at the $1 \%$ level. The same pattern obtains if

\footnotetext{
${ }^{18}$ Sorting by $\triangle$ VIX is performed across all announcement days within the respective group of macro indicators. As a result, the number of events in the high-uncertainty group is 15 for NFP, 51 for ISM, 19 for GDP and 78 for FOMC, with lower numbers for NFP and GDP because their announcements occur at 8:30 am outside of the regular trading hours and thus have fewer observations of VIX. Our results, however, are not dominated by a particular type of announcements, such as FOMC or ISM. Sorting within each announcement type, the results remain robust.
} 


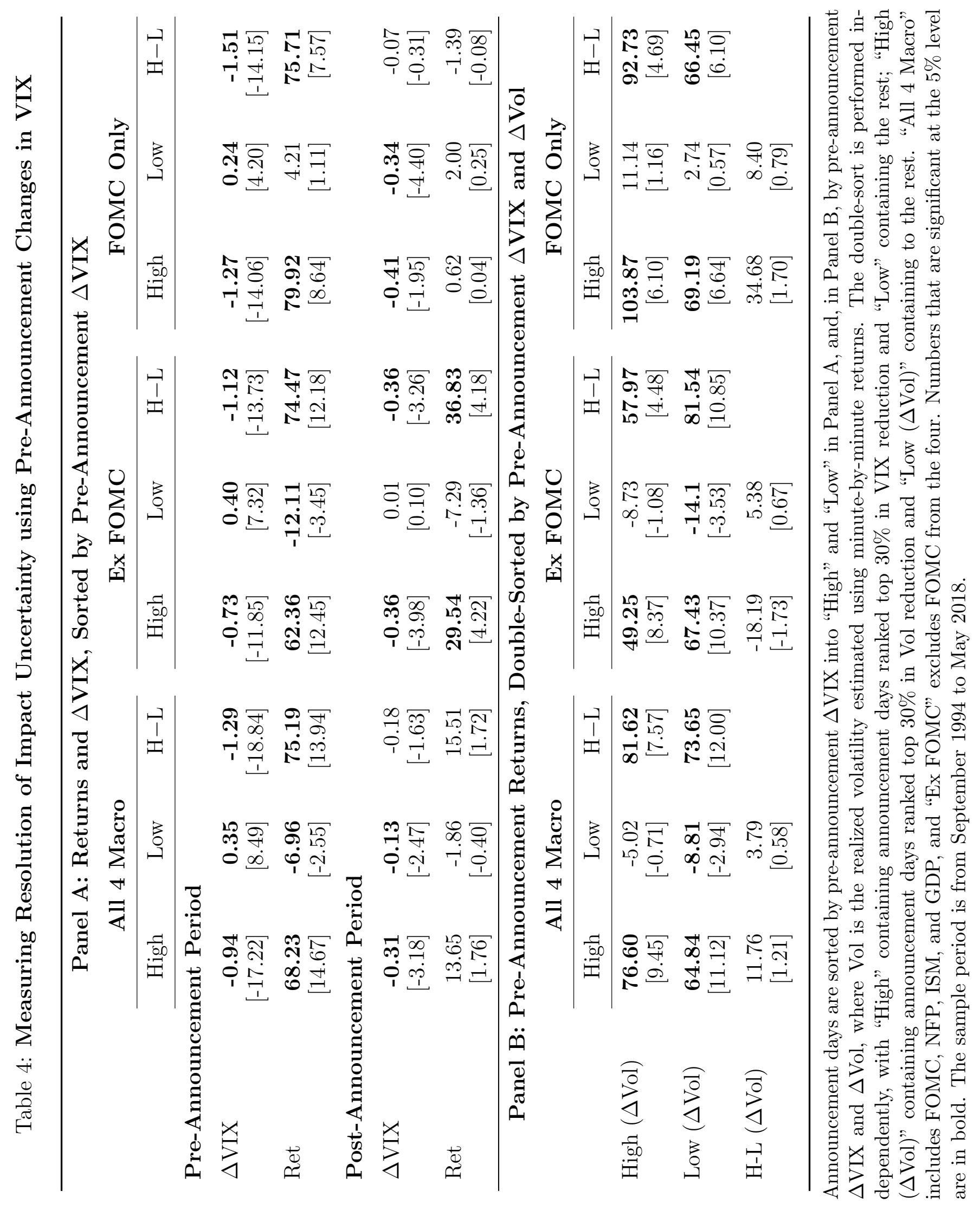


we restrict attention to Macro excluding FOMC or FOMC only.

Given the well established contemporaneous relation between return and changes in return volatility, it is important for us to differentiate the return-VIX relation from that of return-volatility. For this, we further use intraday minute-by-minute returns on E-mini S\&P 500 index futures to estimate the realized volatility. To capture the pre-announcement changes in realized volatility, we subtract the realized volatility of the pre-announcement window by its counterpart on the previous trading day, which the realized volatility calculated as the square root of the sum of squared log returns on E-mini S\&P 500 index futures sampled at 1-minute frequency. We then assign announcement days into four independently sorted groups, by change of VIX $(\Delta$ VIX) and change of realized volatility $(\Delta \mathrm{Vol})$ during the pre-announcement period. High groups contain announcements with the largest $30 \%$ reduction in VIX or realized volatility, and low groups contain the rest of the announcements. As reported in Panel B of Table 4, the difference in pre-announcement return across the $\Delta$ VIX dimension remains rather robust, with magnitudes close to the findings in Panel $\mathrm{A}$, for both the high and low $\Delta \mathrm{Vol}$ groups. By contrast, across the $\Delta \mathrm{Vol}$ dimension, we do not observe any significant difference in pre-announcement return. This contrast between VIX and realized volatility confirms our model's prediction with respect to VIX and $\lambda$. In particular, as stated in Proposition 2, the reduction in the equilibrium price of the variance swap (i.e., $V I X^{2}$ ) during the pre-announcement period, reflecting the resolution of impact uncertainty, increases with the magnitude of impact uncertainty $\lambda$.

For the post-announcement period, our results are relatively weak. Focusing first on post-announcement returns, Panel A of Table 4 shows that only for the three macroeconomic announcements (Macro ex FOMC) do we see significant post-announcement returns in the high uncertainty group, of 29.54 basis points, which is quite large but still substantially lower than the 62.36 basis points observed for pre-announcement return. For the four macroeconomic announcements, the post-announcement return is 13.65 basis points in the high uncertainty group, with a marginal t-stat of 1.76. Focusing next on the dynamics of VIX, we see that VIX continues to decline post-announcement for both high- and lowuncertainty groups. For high uncertainty groups, the magnitude of the decline is about one-third to one-half of the pre-announcement counterparts, indicating that uncertainty is resolved mostly before the announcements. For low uncertainty groups, VIX declines slightly after announcements, as opposed to increasing before the announcements. The timing of the uncertainty resolution, as captured by the dynamics of VIX, is therefore quite different for 


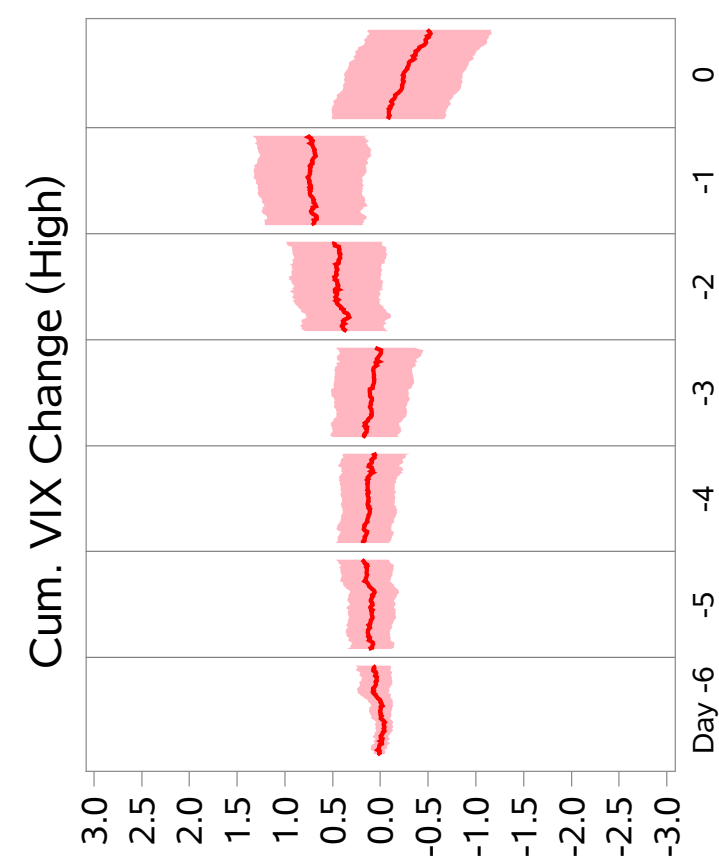

(\%) әธธечว XI^ 'unว

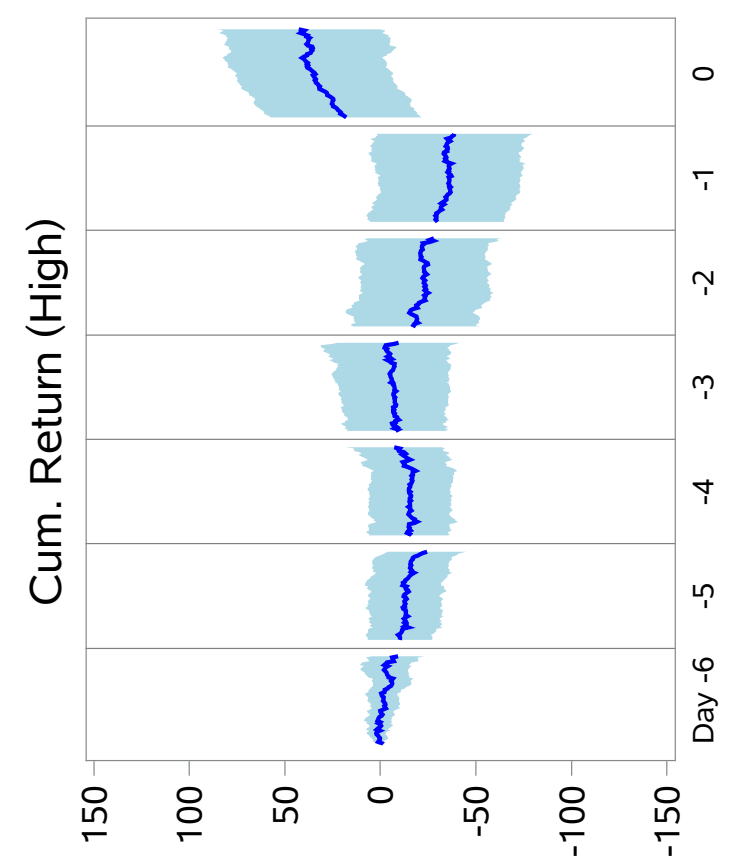

(sdq) fəy 'unכ

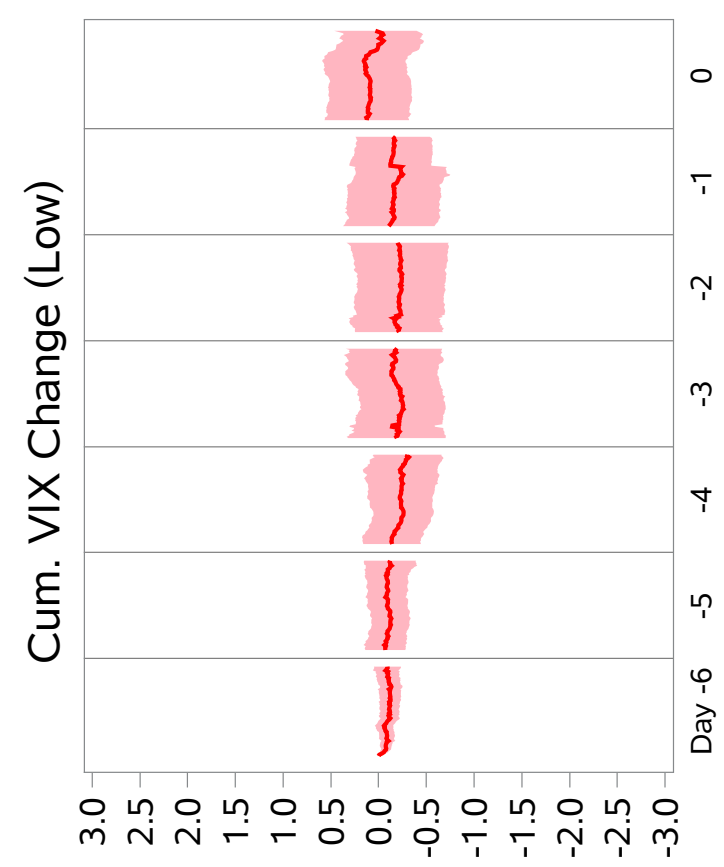

(\%) әБиечว XI^ 'யนnว

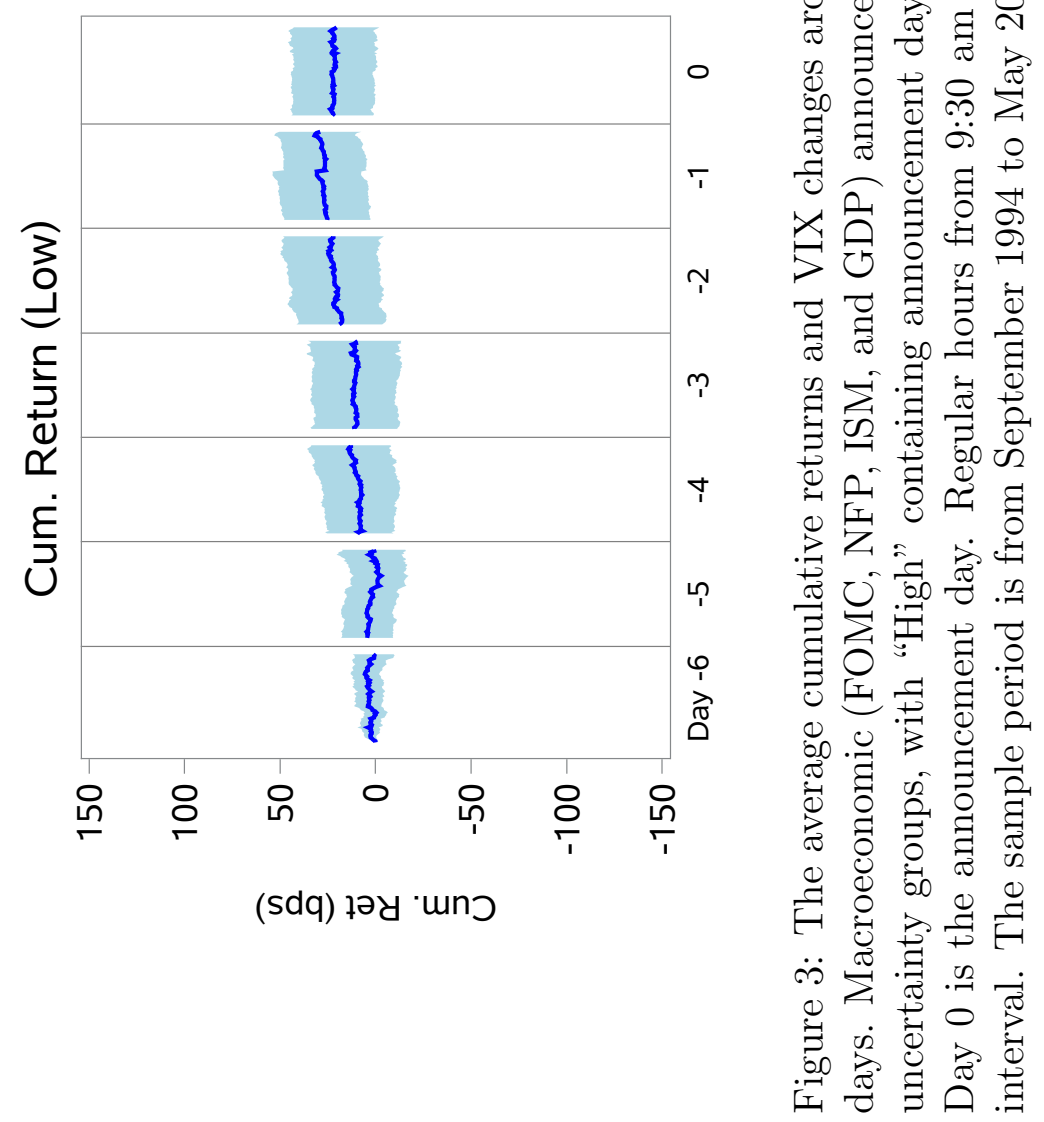


high- and low-uncertainty groups.

Finally, Figure 3 plots the pattern of return and $\Delta$ VIX surrounding the macroeconomic announcements for both the high and low uncertainty groups. Contrasting the patterns between the two group serves as a graphical illustration of the key mechanism of our model. With heightened uncertainty, the high-uncertainty group serves as a "turbo" version of the average results and paints a sharper picture of our model predictions. During the accumulation period, as a result of heightened uncertainty, we observe a gradual rise in VIX, accompanied with a gradual decline in stock price. Immediately before the announcement, mostly during the overnight period before the market open, we observe a sharp drop in VIX and a large rise of stock prices, as a result the resolution of impact uncertainty. By contrast, the low-uncertainty group does not exhibit such patterns. In particular, the gradual decline in stock price and build-up in VIX are missing for the low-uncertainty group during the accumulation period.

\subsection{Heightened Uncertainty and Its Risk Premium}

Prediction 4 of our model proposes the build-up of VIX during the accumulation period as another alternative measure of the magnitude of impact uncertainty. Varying across different announcement days, the higher the impact uncertainty, the larger the build-up of VIX during the accumulation period. The empirical challenge, however, is that we do not know exactly when uncertainty starts to build up, which may vary substantially across announcements. Using any fixed window across all announcements inevitably introduces noises in our measurement and weakens our results. Moreover, the pre-scheduled nature of macroeconomic releases allows investors to trade well in advance, which in turn masks the real market impact over a relatively long time window.

In our empirical tests, we measure the VIX build-up over a six-day accumulation period. Specifically, the accumulation-period $\Delta$ VIX is measured by $\operatorname{VIX}_{t-1}-\mathrm{VIX}_{t-7}$, using information up to the day before the announcement day $t$. Using this accumulation-period $\Delta$ VIX, we sorting announcement days into high and low uncertainty groups, with the high group containing days with accumulation-period $\triangle$ VIX in the top 30 percentile of the sample, and the low group containing the rest. As reported in Table 5, our analysis is performed over three groups of macroeconomic indicators, with the most comprehensive analysis including all four of the macroeconomic indicators (NFP, ISM, GDP and FOMC). ${ }^{19}$

\footnotetext{
${ }^{19}$ Under this specification, the high uncertainty group contains a balanced set of announcement days from
} 


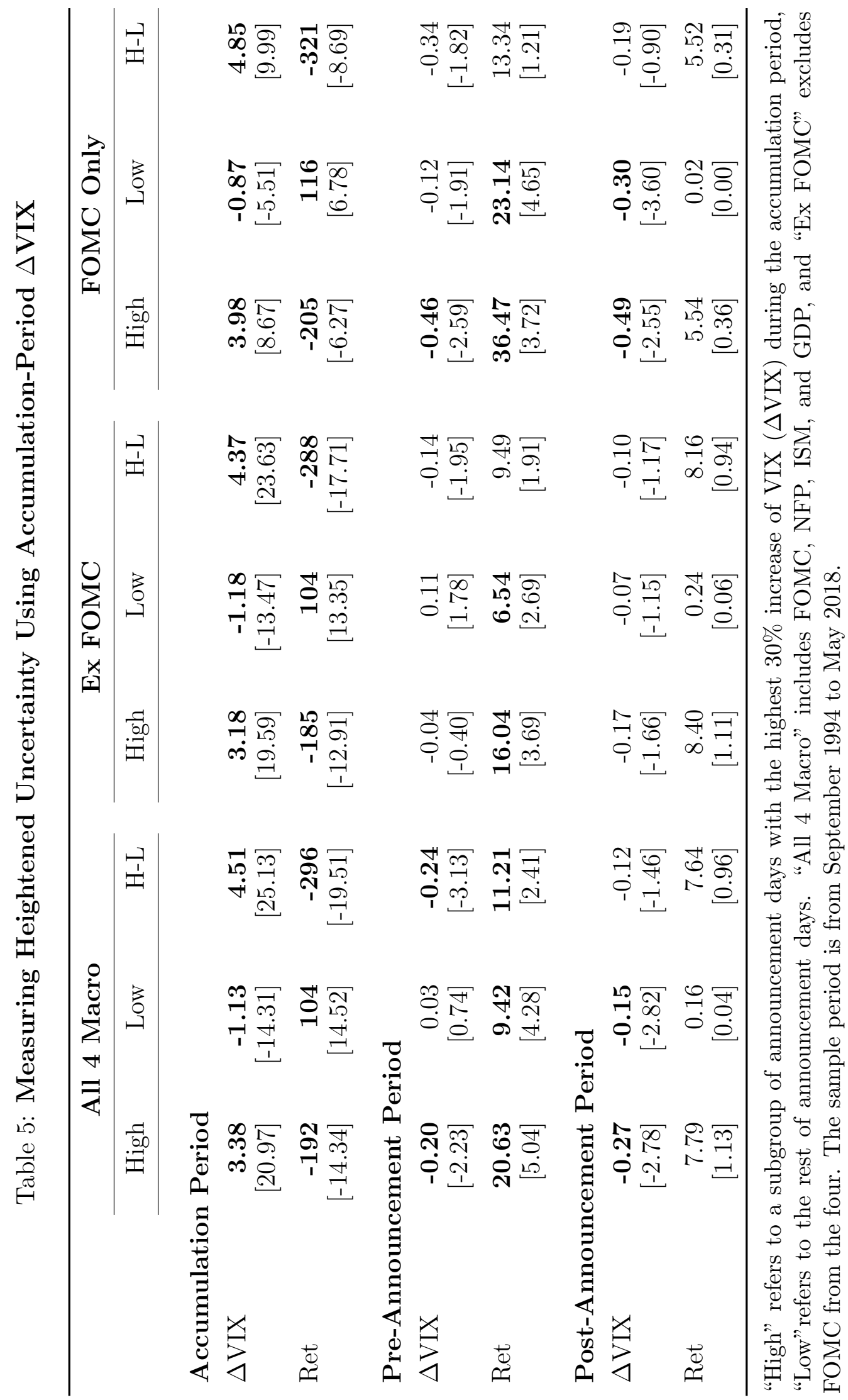


Focusing first on the accumulation period, we see that, by construction, there is a large VIX build-up for the high uncertainty group during the accumulation period. Using all four macroeconomic indicators, the average $\triangle$ VIX is 3.38 percentage points for the high group, and associated with the VIX spikes are large negative returns of -192 basis points over the six-day accumulation period. For the low-uncertainty group of events, VIX declines by 1.13 percentage points and the associated market return is $104 \mathrm{bps}$. So far, we've constructed two groups of announcement days exhibiting distinctively different patterns in $\Delta$ VIX and return during the accumulation period. In particular, the announcement days in the high group are unique in their substantial build-up in VIX, accompanied by large negative return.

Following these two groups into the pre-announcement period, we find rather interesting empirical results that are consistent with Prediction 4 of our model. As shown in Table 5, the average pre-announcement return is 20.63 basis points for the high group, statistically significant and twice as large the 9.42 basis points for the low group. Consistent with Prediction 4 of our model, the high group, which contains announcement days of heightened uncertainty, yields higher pre-announcement returns. Also consistent with the prediction, we find significant decline of the pre-announcement of VIX, reflecting the resolution of impact uncertainty, only for the high group. As shown in Table 5, the average pre-announcement change in VIX is -0.20 percentage points and statistically significant for the high group, and 0.03 percentage points and statistically insignificant for the low group. Table 5 further tests the differences in pre-announcement returns as well $\Delta$ VIX between the high and low groups, and find the differences to be statistically significant.

For the post announcement period, returns are in general insignificant. The average post-announcement returns are approximately 8 basis points for high uncertainty group of events. Though not significant statistically, the magnitudes are still larger than those of low uncertainty, which are close to zero basis points on average. VIX continues to decline after announcements, slightly more so for high uncertainty events.

In addition to the comprehensive test using all four macroeconomic indicators, Table 5 also reported the tests using the three macroeconomic indicators (NFP, ISM, and GDP) as well as using the FOMC only. Overall, the empirical results paint a rather consistent story, confirming Prediction 4 of the model. Since heightened uncertainty is measured during the accumulation period which takes place before the pre-announcement period, this result

the four indicators, with 80 announcement days for NFP, 93 for ISM, 77 for GDP, and 51 for FOMC. In other words, our results are not dominated by any subset of the indicators and sorting within each indicator yields robust results. 
casts independent support for the two-risk explanations for the pre-announcement return premium.

Table 6: Predicting Pre-Annoucement Returns by Accumulation-Period $\Delta$ VIX

\begin{tabular}{|c|c|c|c|c|c|c|}
\hline & \multicolumn{2}{|c|}{ All 4 Macro } & \multicolumn{2}{|c|}{ Ex FOMC } & \multicolumn{2}{|c|}{ FOMC Only } \\
\hline & Ret & $\Delta \mathrm{VIX}$ & Ret & $\Delta \mathrm{VIX}$ & Ret & $\Delta \mathrm{VIX}$ \\
\hline Constant & $\begin{array}{l}12.86^{* * *} \\
{[6.59]}\end{array}$ & $\begin{array}{c}-0.05 \\
{[-1.22]}\end{array}$ & $\begin{array}{l}9.15^{* * *} \\
{[4.28]}\end{array}$ & $\begin{array}{c}0.06 \\
{[1.13]}\end{array}$ & $\begin{array}{c}27.14^{* * *} \\
{[5.98]}\end{array}$ & $\begin{array}{c}-0.22^{* * *} \\
{[-3.25]}\end{array}$ \\
\hline$\Delta \operatorname{VIX}[-6,-1]$ & $\begin{array}{l}3.40^{* * *} \\
{[2.79]}\end{array}$ & $\begin{array}{c}-0.07^{* *} \\
{[-2.57]}\end{array}$ & $\begin{array}{c}3.51^{* *} \\
{[2.34]}\end{array}$ & $\begin{array}{c}-0.07^{* *} \\
{[-2.00]}\end{array}$ & $\begin{array}{c}2.48^{*} \\
{[1.71]}\end{array}$ & $\begin{array}{c}-0.06 \\
{[-1.51]}\end{array}$ \\
\hline Adj R-Sqr (\%) & $\begin{array}{r}3.13 \\
022\end{array}$ & 5.50 & $\begin{array}{r}3.38 \\
722\end{array}$ & $\begin{array}{r}5.46 \\
222\end{array}$ & 1.16 & 4.17 \\
\hline
\end{tabular}

Returns and changes in VIX during the pre-announcement period are regressed on lagged changes in VIX during the accumulation period. The regressands are demeaned so that the intercept reflects the average event day returns and $\triangle$ VIX. Returns are in basis points and $\Delta$ VIX are in percent. "All 4 Macro" includes FOMC, NFP, ISM, and GDP, and "Ex FOMC" excludes FOMC from the four. The sample period is from September 1994 to May 2018.

To further strengthen this result, which is predictive in nature, we regress pre-announcement returns on $\triangle$ VIX measured in the accumulation period, and report the results in Table 6 . Consistent with Prediction 4 of the model, across all three specifications, accumulationperiod $\Delta$ VIX can positively predict the pre-announcement return. For example, for specification using all four macroeconomic indicators, a one percentage point increase of VIX in the accumulation period leads to a 3.40 basis points increase in pre-announcement return. The adjusted R-squared of the regression is $3.13 \%$, which is rather large for predictive regressions of daily stock returns and a large fraction of the pre-announcement returns in this predictive regression are in fact realized overnight.

\section{Empirical Results: Unanticipated Heightened Uncertainty}

In addition to pre-scheduled announcements, heightened uncertainty can also be triggered unexpectedly. In this section, we expand the implications of our model beyond the scheduled announcements to include such unanticipated heightened uncertainty. According to Prediction 5 of our model, following unanticipated heightened uncertainty, there should be reversals in VIX and positive stock returns as the uncertainty resolves. Effectively, this exercise serves as an out-of-the-sample test of the key mechanism of our model by showing that heightened uncertainty, regardless of its origin, brings risk as well as risk premium to the market. 


\subsection{Capturing Unanticipated Heightened Uncertainty}

To capture episodes of unanticipated heightened uncertainty, we take advantage of our model's prediction, which yields changes in VIX as a gauge of impact uncertainty. In the context of our model, a large spike in VIX on a single day can be viewed as a condensed, spedup version of the slow accumulation of VIX in anticipation of a scheduled announcement. Following this observation, we focus mainly on daily changes in VIX, defined by

$$
\Delta \mathrm{VIX}_{t}=\mathrm{VIX}_{t}-\mathrm{VIX}_{t-1}
$$

Our data sample for studying surprise VIX hikes is larger, starting in January 1986 and ending in May 2018. For the early period from 1986 through 1989, we use the "old VIX" index (VXO), and after that we use the current form of VIX index. To compare with macroeconomic announcements, we also report results for the sub-period from September 1994 to May 2018.

Over the full sample, $\Delta \mathrm{VIX}_{t}$ has a slightly negative but insignificant mean, and a standard deviation of $2.16 \%$. The events surrounding the 1987 stock market crash significantly affect the distribution of $\Delta \mathrm{VIX}_{t}$, resulting in extreme values in its skewness and kurtosis. Taking out October 1987, the sample standard deviation of $\Delta \mathrm{VIX}_{t}$ is $1.51 \%$, skewness is close to 1 (with a t-stat of 2.77), and kurtosis is 24 (with a t-stat of 6.09). Overall, the distribution of $\Delta$ VIX $_{t}$ is marked by large movements in the tails, with sudden spikes in VIX being more frequent and larger in magnitude than sudden reductions in VIX. Our objective in this section is to use the tail events associated the sudden spikes in VIX to capture heightened uncertainty in financial markets and measure the premium for heightened uncertainty.

At the close of trading day $t$, we define day $t+1$ as a heightened VIX day, if $\Delta \mathrm{VIX}_{t}$ is larger than a pre-determined constant cutoff value. As shown in Table 7 , different cutoff values allow us to focus on the different parts of the tail distribution of $\Delta \mathrm{VIX}_{t}$, with higher cutoff resulting in fewer heightened VIX days. For the post-1994 sample period, a cutoff value of $2.5 \%$ yields an average of 11.1 heightened VIX days per year, comparable to the monthly frequency of NFP, ISM, and GDP, while a higher cutoff value of $3.0 \%$ results in an average of 7.7 heightened VIX days per year, comparable to the FOMC frequency. Rather interestingly, the corresponding next-day returns, as reported under $\operatorname{Ret}_{t+1}$ in Table 7, are 36.59 and 42.70 basis points, respectively, and both statistically significant. This result confirms the prediction of our model: following heightened uncertainty, the stock market experiences positive and significant return. Moreover, the higher the uncertainty, the stronger 


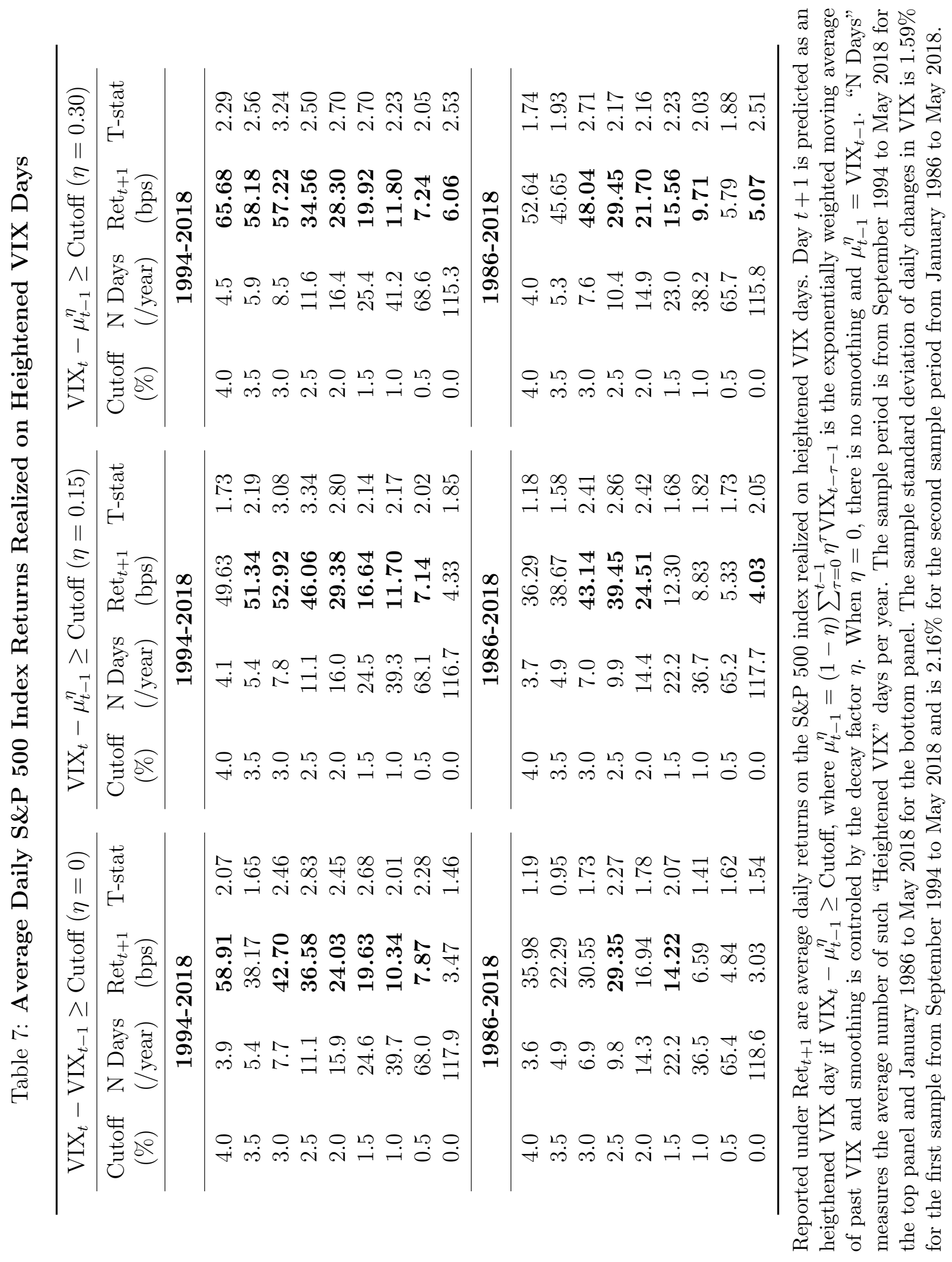


the risk premium.

To further improve our results and smooth out the potential noise in daily changes in VIX, we compare the level of VIX relative to its exponentially weighted moving average. Specifically, at the close of trading day $t$, we define day $t+1$ as a heightened VIX day if

$$
\mathrm{VIX}_{t}-\mu_{t-1}^{\eta} \geq \mathrm{cutoff}
$$

where

$$
\mu_{t-1}^{\eta}=(1-\eta) \sum_{\tau=0}^{t-1} \eta^{\tau} \operatorname{VIX}_{t-\tau-1}
$$

with $\eta$ serving as the decay factor. When $\eta=0$, there is no smoothing, $\mu_{t-1}^{\eta}=\mathrm{VIX}_{t-1}$, and we are back to the simple $\Delta \mathrm{VIX}_{t}$ version.

As shown in the right two panels of Table 7, smoothing the past VIX helps improve our results, especially for the early sample that includes the late 1980s. For example, keeping the same cutoff value of $3 \%$ as before, smoothing the past VIX with a decay factor of $\eta=0.30$ yields an average of 7.6 heightened VIX days per year, comparable to the FOMC frequency. And the average return realized on such heightened VIX days is 48.04 basis points and significant. Using the exponentially weighted moving average of past VIX to select heightened VIX days is equivalent to assuming an $\operatorname{ARIMA}(0,1,1)$ model for the VIX process. For the sample from January 1986 to May 2018, $\eta$ is estimated to be 0.14 with a standard error of 0.01 . We therefore also report the results based on $\eta=0.15$ in Table 7 . Overall, it should be emphasized that our results are robust to a wide range of values of $\eta$.

Given the close connection among return, variance, and VIX, it is natural to ask whether heightened uncertainty can be captured by sudden drops in price or sudden increase in variance. In the context of our model, this is not case, as the model yields VIX as a distinctive empirical measure for the magnitude of the impact uncertainty, while links the magnitude of the news risk to return variance. This implication of our model is confirmed empirically in Table 8, which examines these possibilities. As shown in the left panel, after large price drops, the stock market does on average yield positive returns on the next day, but the predicted returns are statistically insignificance. In other words, although the correlation between daily returns and daily changes in VIX is close to $-70 \%$, the information contents of these two variables are not the same when it comes to capturing heightened uncertainty. The right panel of Table 8 examines the ability of realized variance to capture heightened uncertainty. After large increase in realized volatility, computed using intraday 5-minute 
Table 8: Days After Large Changes in Returns and Intraday Volatility

\begin{tabular}{|c|c|c|c|c|c|c|c|}
\hline $\begin{array}{c}\text { Cutoff } \\
(\%)\end{array}$ & $\begin{array}{l}\text { N Days } \\
\text { (/year) }\end{array}$ & $\begin{array}{l}\text { Ret } \\
\text { (bps) }\end{array}$ & T-stat & $\begin{array}{c}\text { Cutoff } \\
(\%)\end{array}$ & $\begin{array}{l}\text { N Days } \\
\text { (/year) }\end{array}$ & $\begin{array}{l}\text { Ret } \\
\text { (bps) }\end{array}$ & T-stat \\
\hline \multicolumn{4}{|c|}{ Daily Returns } & \multicolumn{4}{|c|}{ Intraday Volatility } \\
\hline-2.4 & 5.3 & 27.96 & 1.26 & 16 & 4.4 & -26.18 & -1.04 \\
\hline-2.3 & 6.0 & 16.93 & 0.85 & 15 & 5.2 & -18.32 & -0.85 \\
\hline-2.2 & 7.0 & 14.70 & 0.84 & 14 & 5.8 & -19.24 & -0.96 \\
\hline-2.1 & 7.7 & 12.15 & 0.74 & 13 & 6.9 & -17.05 & -1.00 \\
\hline-2.0 & 8.6 & 12.68 & 0.86 & 12 & 8.3 & -18.12 & -1.24 \\
\hline-1.9 & 9.5 & 19.23 & 1.40 & 11 & 9.6 & -17.39 & -1.32 \\
\hline-1.8 & 11.1 & 15.37 & 1.27 & 10 & 11.2 & -13.32 & -1.14 \\
\hline-1.7 & 12.6 & 17.85 & 1.65 & 9 & 13.5 & -6.82 & -0.68 \\
\hline-1.6 & 14.2 & 14.16 & 1.45 & 8 & 16.6 & -3.75 & -0.44 \\
\hline-1.5 & 16.3 & 15.48 & 1.77 & 7 & 20.5 & 0.77 & 0.11 \\
\hline
\end{tabular}

Daily returns on the S\&P 500 index are used. Intraday volatility is measured using 5-minute S\&P 500 index returns and converted to annual volatility. The heightened uncertainty days are picked if daily returns fall below the "Cutoff" values or daily changes in volatility increase above the "Cutoff" values. "N Days" measures the average number of such extreme days per year. The sample is from January 1986 to May 2018. The sample standard deviations are $1.13 \%$ and $9.42 \%$, respectively, for daily returns and daily changes in volatility.

returns on S\&P 500 index futures, the next-day returns are on average negative but statistically insignificant. In other words, although the daily correlation between VIX and realized volatility is as high as $77 \%$, they contain very different information for the purpose of capturing heightened uncertainty and predicting returns. In particular, the volatility component in VIX is not helpful for this purpose.

\subsection{A Common Mechanism: Heightened Uncertainty and Its Resolution}

This section draws a direct comparison between the anticipated and unanticipated heightened uncertainty. In particular, we hypothesize that the underlying mechanism that drives the join dynamics of return and uncertainty on heightened VIX days is similar to that for prescheduled macroeconomic announcements. To illustrate this common mechanism, we plot the patterns of cumulative returns and VIX around heightened VIX days in Figure 4 and match the timing to that for the scheduled announcements. In particular, we label the day when VIX spikes up as day -1, which is a sped-up version of the six-day accumulation period. We label the heightened VIX days as day 0, parallel to the pre-announcement window when the resolution of impact uncertainty takes place. The heightened VIX days plotted in 


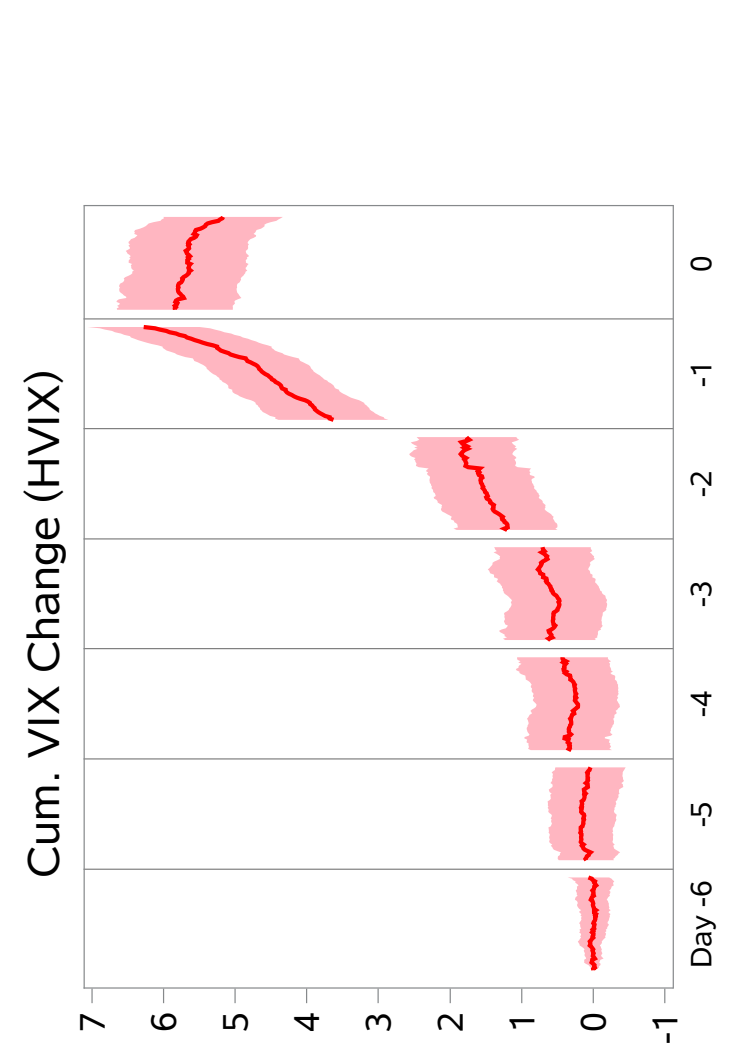

(\%) әธиечว XI^ 'unว

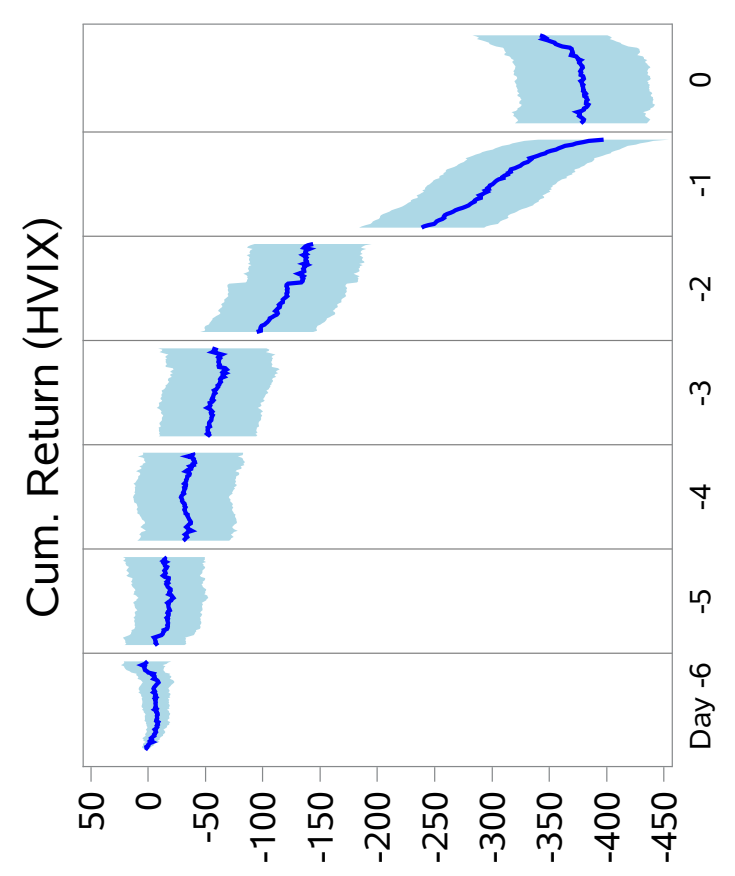

(sdq) fəy 'unכ

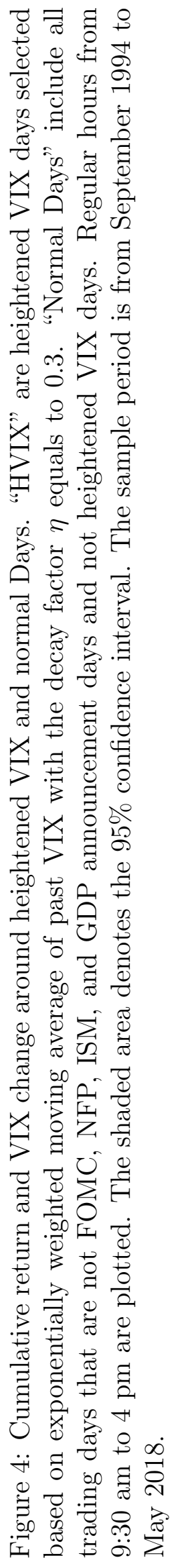


Figure 4 are selected based on exponentially weighted moving average of past VIX with the decay factor $\eta$ equals to 0.3 , although the pattern is robust to different choices of values for the decay factor $\eta$.

The top panel of Figure 4 shows that stock markets drops 389 basis points from day -6 to day -1 . Associated with the stock price decline is a staggering increase of VIX by 6.28 percentage points. Recall from Table 5 that in the high uncertainty group of scheduled macroeconomic announcements, VIX increases by 4.29 percentage points and stock return declines by about 255 basis points. These numbers are in the same order of magnitude, although HVIX events are more dramatic. The bottom panel of Figure 4 shows that on normal days, there is barely any change in VIX and stock prices slowly drift up at a rate consistent with the annualized stock returns. Overall, the evidence suggests that the returns and VIX changes around scheduled macroeconomic announcements and those around HVIX days are qualitatively and quantitatively similar.

Table 9: Predicting Non-Announcement Day Return and $\Delta$ VIX by VIX Build-up

\begin{tabular}{|c|c|c|c|c|}
\hline & \multicolumn{2}{|c|}{ HVIX } & \multicolumn{2}{|c|}{ Normal Days } \\
\hline & Ret & $\Delta \mathrm{VIX}$ & Ret & $\Delta \mathrm{VIX}$ \\
\hline Constant & $\begin{array}{l}57.22^{* * *} \\
{[3.90]}\end{array}$ & $\begin{array}{l}-1.11^{* * *} \\
{[-4.47]}\end{array}$ & $\begin{array}{c}-0.85 \\
{[-0.52]}\end{array}$ & $\begin{array}{l}0.09^{* * *} \\
{[4.32]}\end{array}$ \\
\hline$\Delta \operatorname{VIX}[-6,-1]$ & $\begin{array}{c}7.03^{*} \\
{[1.75]}\end{array}$ & $\begin{array}{l}-0.15^{* * *} \\
{[-2.69]}\end{array}$ & $\begin{array}{c}0.33 \\
{[0.41]}\end{array}$ & $\begin{array}{c}-0.02^{* *} \\
{[-2.01]}\end{array}$ \\
\hline Adj R-Sqr (\%) & 2.34 & 4.76 & -0.01 & 0.21 \\
\hline Obs & 202 & 202 & 4814 & 4814 \\
\hline
\end{tabular}

Daily returns on the S\&P 500 index and changes in VIX are regressed on lagged changes in VIX over a six-day window. "HVIX" are heightened VIX days selected based on exponentially weighted moving average of past VIX with the decay factor $\eta$ equals to 0.3. "Normal Days" include all trading days that are not FOMC, NFP, ISM, and GDP announcement days and not heightened VIX days. The regressands are demeaned so that the intercept reflects the average event day returns and $\Delta$ VIX. Returns are in basis points and $\Delta$ VIX are in percent. The reported t-stat's use Newey-West standard errors, adjusting for serial correlations. The sample period is from September 1994 to May 2018.

The similarity between heightened VIX events and scheduled macroeconomic announcements are also evident in Table 9, where we regress stock returns and VIX changes on day 0 (HVIX days) on the change of VIX from day -7 to day -1 . Among the HVIX events, a higher VIX increase predicts a higher stock return and a lower VIX. A one percent higher VIX increase from day -7 to day -1 predicts a 7.03 bps higher stock return and a $0.15 \%$ 
decline in VIX on day 0. The statistical significance of these coefficients is borderline, but the signs are the same as those of pre-scheduled announcements.

By comparison, we run the same regression on normal days which are not scheduled NFP, ISM, GDP and FOMC announcement days and not associated with heightened VIX days. The coefficient is 0.33 and statistically insignificant for return; -0.02 and marginal significant for $\triangle$ VIX. On normal days, which do not bring heightened uncertainty to the market by construction, the predicative power of VIX buildup is much weaker in both statistical significance and economic magnitudes.

\section{Conclusion}

We find that the U.S. stock market earns a large overnight return right before important macroeconomic announcements, including NFP, ISM, and GDP, with no increase in conventional risk measures. The large pre-announcement returns are qualitatively and quantitatively similar to the pre-FOMC announcement drift documented by Lucca and Moench (2015). Moreover, for all four macroeconomic announcements, the pre-announcement returnto-variance ratio far exceeds the post-announcement counterparts, strongly suggesting that distinct types of risk premia are realized around the announcement window.

To explain this common pattern, we propose a two-risk model in which, in addition to the directional news risk itself, uncertainty about the magnitude of its market impact is the second risk. Importantly, this impact uncertainty is resolved before the news risk itself is fully resolved at the announcement. In the model, VIX emerges as a measure of impact uncertainty and return variance gives the standard measure of news risk. The model then generates the empirical pattern that pre-announcement return and return-to-risk ratio are much larger than their post-announcement counterparts. Moreover, the model yields clear predictions on the joint dynamics of return, variance and VIX surrounding an announcement. In particular, heightening of uncertainty concerning an announcement is captured by a rise in VIX in the accumulation period; it brings a contemporaneous price drop, and leads to a high pre-announcement drift accompanied by a large drop in VIX, reflecting the resolution of the impact uncertainty. Using the overnight period right before the announcement as the pre-announcement period and several days prior as the accumulation period, we empirically confirm all these predictions. Finally, analogous to the anticipated rise of uncertainty ahead of macroeconomic announcements, we find that large surprise spikes of VIX are also followed by higher stock returns and VIX reversals in the next day. 
In sum, the evidence strongly suggest that heightened uncertainty about the impact of important macroeconomic announcements is a useful theme for organizing the facts about returns, risks, and return-to-risk tradeoffs in the stock market around such news events. The large risk premiums these announcements bring to the market and its fast realization raise interesting questions about the underlying mechanism driving such a process, its impact on investors, and potential alternative designs for the production and release process of the announcement information. ${ }^{20}$ We leave these questions for future research.

\footnotetext{
${ }^{20}$ To the extent that the resolution of uncertainty is a result of learning, a possible direction for future research is to use granular, micro-level data to uncover investors' behavior around these announcements. For example, using clicks on news articles, Benamar, Foucault, and Vega (2020) find strong evidence of learning by investors in the overnight period before nonfarm payroll releases. Since the release process of macroeconomic announcements is often endogenous, its design can significantly affect the informational efficiency of the market. As an example, $\mathrm{Hu}, \mathrm{Pan}$, and Wang (2017) examines how tiered release of macroeconomic indicator such as ISM can substantially impact the price discovery process.
} 


\section{Appendix A: Proofs}

Equilibrium Price at $t=0$

Let $J_{0}=E\left[J_{1}\right]$. Then,

$$
\begin{aligned}
\frac{d J_{0}}{d \theta_{0}}= & -e^{-\alpha\left[W_{0}+\theta_{0}\left(\bar{D}-P_{0}\right)\right]-\alpha^{2} Q\left(\theta_{0}, \delta\right)} \frac{1}{1+\alpha^{2} \lambda Q\left(\theta_{0}, \delta\right)} \\
& \cdot\left\{-\alpha\left(\bar{D}-P_{0}\right)+\alpha^{2}\left(1-\delta+\delta \theta_{0}\right) \lambda_{0}+\frac{\alpha^{2} \lambda\left(1-\delta+\delta \theta_{0}\right)}{1+\alpha^{2} \lambda Q\left(\theta_{0}, \delta\right)}\right\}
\end{aligned}
$$

where $Q\left(\theta_{0}, \delta\right)$ is given by (11). Setting the above expression to zero and substituting in $\theta_{0}=1$, we obtain (15). It is easy to check that the second-order condition holds, i.e., $\frac{d^{2} J_{0}}{d \theta_{0}^{2}}<0$. In particular, write $d J_{0} / d \theta_{0}=J_{0} K$, where $K$ is the expression in the curly bracket of (A.1). Then $d^{2} J_{0} / d \theta_{0}^{2}=\left(d J_{0} / d \theta_{0}\right) K+J_{0} d K / d \theta_{0}=J_{0} K^{2}+J_{0} d K / d \theta_{0}$. Note that $K$ is strictly increasing in $\theta_{0}$ and $J_{0}<0$. So $d^{2} J_{0} / d \theta_{0}^{2}<0$.

\section{Mean and Variance of Returns}

From the stock prices at $t=0,1$ given in (15) and (8), the mean of the return in period 1 is:

$$
\mathrm{E}\left[R_{1}\right]=\alpha \lambda_{0}+\frac{\alpha \lambda}{1-\frac{1}{2} \alpha^{2} \lambda}-\alpha(1-\delta) \mathrm{E}\left[\sigma^{2}\right]=\delta \alpha\left(\lambda_{0}+\lambda\right)+\frac{\frac{1}{2} \alpha^{3} \lambda^{2}}{1-\frac{1}{2} \alpha^{2} \lambda}>0,
$$

where we have used the fact that $\mathrm{E}\left[\sigma^{2}\right]=\lambda_{0}+\lambda$. The variance of the return is:

$$
\begin{aligned}
\mathrm{V}\left[R_{1}\right] & =\mathrm{V}\left[\sigma \varepsilon_{1}-\alpha(1-\delta) \sigma^{2}\right]=\mathrm{E}\left[\left[\sigma \varepsilon_{1}-\alpha(1-\delta) \sigma^{2}\right]^{2}\right]-\left(\mathrm{E}\left[\sigma \varepsilon_{1}-\alpha(1-\delta) \sigma^{2}\right]\right)^{2} \\
& =\delta\left(\lambda_{0}+\lambda\right)+\alpha^{2}(1-\delta)^{2} \lambda^{2}
\end{aligned}
$$

Likewise, the mean and variance of the return over the second period are:

$$
\begin{aligned}
\mathrm{E}\left[R_{2}\right] & =\mathrm{E}\left[\sigma \varepsilon_{2}+\alpha(1-\delta) \sigma^{2}\right]=\alpha(1-\delta)\left(\lambda_{0}+\lambda\right)>0, \\
\mathrm{~V}\left[R_{2}\right] & =\mathrm{V}\left[\sigma \varepsilon_{2}+\alpha(1-\delta) \sigma^{2}\right]=\mathrm{E}\left(\left[\sigma \varepsilon_{2}+\alpha(1-\delta) \sigma^{2}\right]^{2}\right)-\left(\mathrm{E}\left[\sigma \varepsilon_{2}+\alpha(1-\delta) \sigma^{2}\right]\right)^{2} \\
& =(1-\delta)\left(\lambda_{0}+\lambda\right)+\alpha^{2}(1-\delta)^{2} \lambda^{2}
\end{aligned}
$$

\section{Deriving Proposition 3}

From the expected returns over period 1 and 2, given in (19) and (20), we can see that for a sufficiently large $\lambda$, in particular when it approaches its upper bound $2 / \alpha^{2}$, the expected return for the first period can be very large. Thus, we want to find a threshold $\lambda^{*}$ such that 
when $\lambda$ exceeds this threshold, the expected return and return to variance ratio for the first period are higher than those for the second period.

First, $\mathrm{E}\left[R_{1}\right]>\mathrm{E}\left[R_{2}\right]$ is equivalent to:

$$
\frac{\frac{1}{2} \alpha^{3} \lambda^{2}}{1-\frac{1}{2} \alpha^{2} \lambda}>(1-2 \delta) \alpha\left(\lambda_{0}+\lambda\right)
$$

Since $\delta \in[0,1]$, a sufficient condition for this inequality to hold is:

$$
\frac{\frac{1}{2} \alpha^{3} \lambda^{2}}{1-\frac{1}{2} \alpha^{2} \lambda}>\alpha\left(\lambda_{0}+\lambda\right)
$$

Next, we explore a sufficient condition for $R V R_{1}>R V R_{2}$. First, we note that

$$
R V R_{2}=\frac{\alpha\left(\lambda_{0}+\lambda\right)}{\left(\lambda_{0}+\lambda\right)+\alpha^{2}(1-\delta) \lambda^{2}}<\alpha
$$

Moreover,

$$
R V R_{1}=\frac{\alpha \delta\left(\lambda_{0}+\lambda\right)+\frac{1}{2} \alpha^{3} \lambda^{2} /\left(1-\frac{1}{2} \alpha^{2} \lambda\right)}{\delta\left(\lambda_{0}+\lambda\right)+\alpha^{2}(1-\delta)^{2} \lambda^{2}} .
$$

A lower bound of $R V R_{1}$ can be obtained by making $\delta$-related terms as small as possible in the numerator and making $\delta$-related terms as large as possible in the denominator. Thus, a sufficient condition for $R V R_{1}>R V R_{2}$ is:

$$
\frac{\frac{1}{2} \alpha^{3} \lambda^{2} /\left(1-\frac{1}{2} \alpha^{2} \lambda\right)}{\left(\lambda_{0}+\lambda\right)+\alpha^{2} \lambda^{2}}>\alpha
$$

Reorganizing the terms gives the following:

$$
\frac{\frac{1}{2} \alpha^{3} \lambda^{2}}{1-\frac{1}{2} \alpha^{2} \lambda}-\alpha^{3} \lambda^{2}>\alpha\left(\lambda_{0}+\lambda\right)
$$

Comparing (A.7) with (A.11), we see that the latter implies the former. Thus, we only need to focus on (A.11), which is equivalent to:

$$
\frac{\lambda\left(\frac{1}{2} \alpha^{4} \lambda^{2}-1\right)}{1-\frac{1}{2} \alpha^{2} \lambda}>\lambda_{0}
$$

Now we impose a lower bound on $\lambda$ :

$$
\frac{\sqrt{2}}{\alpha^{2}}<\lambda<\frac{2}{\alpha^{2}}
$$

where the upper bound is simply (4). Then, for $\lambda$ satisfying (A.13), we have

$$
\frac{\lambda\left(\frac{1}{2} \alpha^{4} \lambda^{2}-1\right)}{1-\frac{1}{2} \alpha^{2} \lambda}=\frac{\frac{1}{2} \lambda\left(\alpha^{2} \lambda+\sqrt{2}\right)\left(\alpha^{2} \lambda-\sqrt{2}\right)}{1-\frac{1}{2} \alpha^{2} \lambda}>\frac{\left(2 / \alpha^{2}\right)\left(\alpha^{2} \lambda-\sqrt{2}\right)}{1-\frac{1}{2} \alpha^{2} \lambda} .
$$


Thus, a sufficient condition for (A.12) is

$$
\frac{\left(2 / \alpha^{2}\right)\left(\alpha^{2} \lambda-\sqrt{2}\right)}{1-\frac{1}{2} \alpha^{2} \lambda}>\lambda_{0}
$$

which holds if

$$
\lambda>\frac{\sqrt{2} / \alpha^{2}+\lambda_{0} / 2}{1+\alpha^{2} \lambda_{0} / 4}=\frac{\sqrt{2}}{\alpha^{2}}+\frac{(2-\sqrt{2}) \lambda_{0}}{4+\alpha^{2} \lambda_{0}} \equiv \lambda^{*}\left(\lambda_{0}\right) .
$$

This gives Proposition 3 and $\lambda^{*}\left(\lambda_{0}\right) \in\left[\sqrt{2} / \alpha^{2}, 2 / \alpha^{2}\right)$.

\section{Appendix B: Tradeable HVIX Strategies}

One might question whether the HVIX premium can be captured in practice given that the closing time for CBOE's S\&P 500 index options is at 4:15 pm, 15 minutes after the close of the cash market at $4 \mathrm{pm} .^{21}$ To address this concern, we measure daily VIX at 3:30 pm or 3:45 pm, based on CBOE VIX tick data that are available after January 1992. As reported in Table A1, the results based on intraday VIX are similar to those using closing VIX values. For example, for the cutoff value of $3.0 \%$, there are on average 6.5 HVIX days per year using daily changes in VIX measured at $3: 45 \mathrm{pm}$, and 7.0 days per year using daily changes of closing VIX. The average daily returns are very close, 46.94 basis points and 43.32 basis points.

To summarize Table A1, it is possible to use information as early as 3:45 pm to identify the set of days on which heightened uncertainty has been triggered and to invest in U.S. stock markets to capture a large average next-day return. This qualitative pattern is very similar to the evidence for macroeconomic announcements.

\footnotetext{
${ }^{21}$ Using the tick data on the CBOE VIX, we find that pre-2003, the VIX Close is timed at 4pm, and post-2003, the VIX Close is timed at 4:15pm.
} 


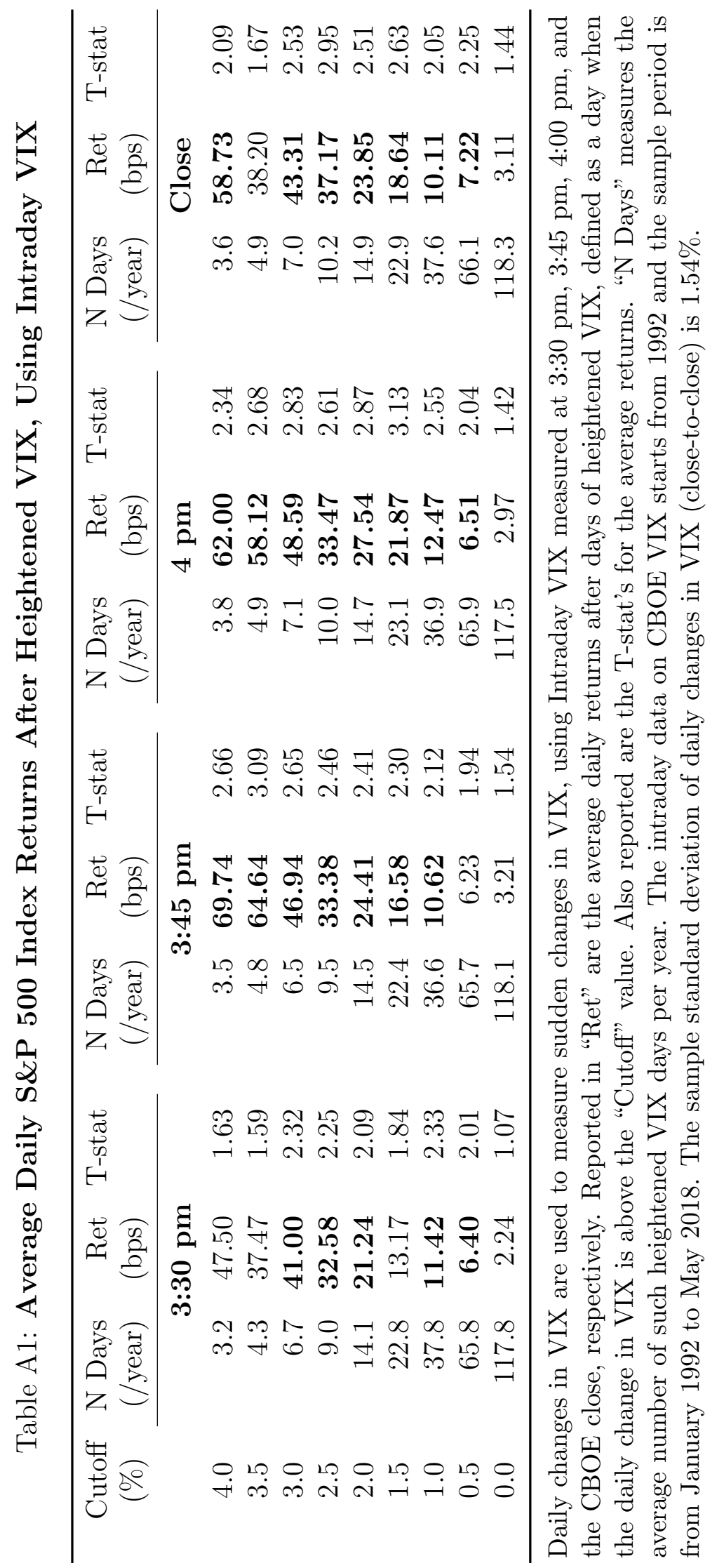




\section{References}

Ai, H. and R. Bansal (2018). Risk Preferences and the Macroeconomic Announcement Premium. Econometrica 86(4), 1383-1430.

Bakshi, G., C. Cao, and Z. Chen (1997). Empirical Performance of Alternative Option Pricing Models. Journal of Finance 52(5), 2003-2049.

Bauer, M., A. Lakdawala, and P. Mueller (2019). Market-Based Monetary Policy Uncertainty. Working paper.

Bekaert, G. and G. Wu (2000). Asymmetric Volatility and Risk in Equity Markets. Review of Financial Studies 13(1), 1-42.

Benamar, H., T. Foucault, and C. Vega (2020). Demand for Information, Macroeconomic Uncertainty, and the Response of U.S. Treasury Securities to News. Review of Financial Studies, Forthcoming.

Bernile, G., J. Hu, and Y. Tang (2016). Can Information be Locked Up? Informed Trading Ahead of Macro-news Announcements. Journal of Financial Economics 121, 496-520.

Black, F. (1976). Studies of Stock Price Volatility Changes. Proceedings of the 1976 Meeting of the Business and Economic Statistics Section, American Statistical Association, Washington DC, 177-181.

Bollerslev, T., G. Tauchen, and H. Zhou (2009). Expected Stock Returns and Variance Risk Premia. Review of Financial Studies 22(11), 4463-4492.

Brusa, F., P. Savor, and M. Wilson (2020). One Central Bank to Rule Them All. Review of Finance 24(2), 263-304.

Campbell, J. and L. Hentschel (1992). No News is Good News: An Asymmetric Model of Changing Volatility in Stock Returns. Journal of Financial Economics 31(3), 281-318.

Carr, P. and L. Wu (2006). A Tale of Two Indices. The Journal of Derivatives 13, 13-29.

Christie, A. A. (1982). The Stochastic Behavior of Common Stock Variances: Value, Leverage and Interest Rate Effects. Journal of Financial Economics 10(4), 407-432.

Cieslak, A., A. Morse, and A. Vissing-Jorgensen (2019). Stock Returns over the FOMC Cycle. Journal of Finance 74(5), 2201-2248. 
Dennis, P., S. Mayhew, and C. Stivers (2006). Stock Returns, Implied Volatility Innovations, and the Asymmetric Volatility Phenomenon. Journal of Financial and Quantitative Analysis 41, 381-406.

Ernst, R., T. Gilbert, and C. Hrdlicka (2019). More Than 100\% of the Equity Premium: How Much is Really Earned on Macroeconomic Announcement Days? Working paper.

Fisher, A., C. Martineau, and J. Sheng (2018). Macroeconomic Attention and the Stock Market. Working paper.

Fleming, M. and M. Piazzesi (2005). Monetary Policy Tick-by-Tick. Working paper.

French, K. R., G. Schwert, and R. F. Stambaugh (1987). Expected Stock Returns and Volatility. Journal of Financial Economics 19(1), 3-29.

Gilbert, T., A. Kurov, and M. Wolfe (2018). The Disappearing Pre-FOMC Announcement Drift. Working Paper.

Guo, R., D. Jia, and X. Sun (2019). Attention, Uncertainty Reduction and Preannouncement Premium in China. Working paper.

Han, B. (2019). Dynamic Information Acquisition and Asset Prices. Working paper.

He, H. and J. Wang (1995). Differential Information and Dynamic Behavior of Stock Trading Volume. The Review of Financial Studies 8(4), 919-972.

Hu, G. X., J. Pan, and J. Wang (2017). Early Peek Advantage? Efficient Price Discovery wiht Tiered Information Disclosure. The Journal of Financial Economics 126, 399-421.

Jiang, G., G. Pan, and Z. Qiu (2019). Pre-announcement Premium. Working paper.

Kurov, A., A. Sancetta, G. Strasser, and M. H. Wolfe (2019). Price Drift before U.S. Macroeconomic News: Private Information about Public Announcements? Journal of Financial and Quantitative Analysis 54(1), 449-479.

Laarits, T. (2020). Pre-Announcement Risk. Working paper.

Lucca, D. and E. Moench (2015). The Pre-FOMC Announcement Drift. Journal of Finance 70(1), 329-371.

Lucca, D. and E. Moench (2018). The Pre-FOMC Announcement Drift: More Recent Evidence. Liberty Street Economics 20181116a, Federal Reserve Bank of New York.

Pan, J. (2002). The Jump-Risk Premia Implicit in Options: Evidence from an Integrated Time-Series Study. Journal of Financial Economics 63(1), 3-50. 
Savaser, T. (2011). Exchange Rate Response to Macronews: Through the Lens of Microstructure. Journal of International Financial Markets, Institutions, and Money 21, $107-126$.

Savor, P. and M. Wilson (2013). How Much Do Investors Care About Macroeconomic Risk? Evidence from Scheduled Economic Announcements. Journal of Financial and Quantitative Analysis 48, 343-375.

Wachter, J. A. and Y. Zhu (2019). A Model of Two Days: Discrete News and Asset Prices. Working paper.

Wu, G. (2001). The Determinants of Asymmetric Volatility. The Review of Financial Studies 14(3), 837-859. 